\title{
Activity-Regulated Dynamic Behavior of Early Dendritic Protrusions: Evidence for Different Types of Dendritic Filopodia
}

\author{
Carlos Portera-Cailliau, David T. Pan, and Rafael Yuste \\ Department of Biological Sciences, Columbia University, New York, New York 10027
}

\begin{abstract}
Dendritic filopodia are long and thin protrusions that occur predominantly during early development of the mammalian CNS. The function of dendritic filopodia is unknown, but they could serve to form early synapses, to generate spines, or to regulate dendritic branching and growth. We used two-photon imaging to characterize the motile behavior of dendritic protrusions during early postnatal development (P2-P12) in pyramidal neurons from acute slices of mouse neocortex. Dendritic protrusions in immature neurons are highly dynamic, and this motility is actin based. Motility and turnover of these early protrusions decreases throughout development, mirroring an increase in their average lifetime and density. Interestingly, density, motility, and length of filopodia are greater in dendritic growth cones than in dendritic shafts. These growth cones disappear after P5. Blocking synaptic transmission globally using TTX or calcium-free solutions led to a $40-120 \%$ increase in the density and length of dendritic filopodia in shafts but not in growth cones. Moreover, blocking ionotropic glutamate receptors resulted in an $\sim 35 \%$ decrease in the density and turnover of shaft filopodia, whereas focal glutamate application led to a $75 \%$ increase in the length of shaft filopodia, but neither manipulation affected growth cone filopodia. Our results support the existence of two populations of filopodia, in growth cones and shafts, which are differentially regulated by neuronal activity. We propose that filopodia in dendritic growth cones are involved in dendritic growth and branching in an activityindependent manner, whereas shaft filopodia are responsible for activity-dependent synaptogenesis and, in some cases, may become dendritic spines.
\end{abstract}

Key words: two-photon; cortex; spine; motility; glutamate; synaptogenesis

\section{Introduction}

Spines were first observed by Ramón y Cajal (1888), who described them as small thorny protrusions on the surface of dendrites of cerebellar Purkinje neurons. He proposed that these spines were the sites of axo-dendritic contact and, therefore, crucial to synaptic transmission (Ramón y Cajal, 1891). In recent years, a virtual explosion in our knowledge about spines has taken place, because of the development of new imaging techniques. In particular, time-lapse imaging of living neurons has demonstrated that spines are very motile and pleomorphic, changing from one morphology to another over a time scale of minutes (Fischer et al., 1998; Dunaevsky et al., 1999; Lendvai et al., 2000). Because of their dynamism, spines appear to exhibit a spectrum of morphologies, so the previously rigid classification systems for spines (Peters and Kaiserman-Abramof, 1970) likely refer to dif-

\footnotetext{
Received Feb. 5, 2003; revised June 9, 2003; accepted June 12, 2003.

This work was supported by National Eye Institute Grant EY13237, the New York Science To Achieve Results Center of the National Center for Environmental Research for High Resolution Imaging of Functional Neural Circuits, the John Merck Fund, and the National Parkinson Foundation. We thank Constantino Sotelo and Kristen Harris for sharing unpublished data, Ayumu Tashiro and Sila Konur for comments, and Huib Mansvelder and Volodymyr Nikolenko for advice on two-photon imaging. We also thank Joshua Sanes and Ania Majewska for providing GFP-M mice for control experiments.

Correspondence should be addressed to Dr. Carlos Portera-Cailliau, Department of Biological Sciences, Columbia University, 1212 Amsterdam Avenue, 1002 Fairchild, New York, NY 10027. E-mail: ccailliau@neuro.columbia.edu. Copyright $\odot 2003$ Society for Neuroscience $\quad$ 0270-6474/03/237129-14\$15.00/0
}

ferent states in a structurally dynamic system (Parnass et al., 2000).

Spines are not the only appendages that protrude from dendrites. During development, dendrites are first decorated by filopodia (Ramón y Cajal, 1934; Morest, 1969; Purpura, 1975; Lund et al., 1977; Vaughn, 1989; Jontes and Smith, 2000; PorteraCailliau and Yuste, 2001). Because of gross similarities between filopodia and spines, information on filopodia has been inferred from existing knowledge about spines. Unfortunately, filopodia are still surprisingly poorly characterized, perhaps because of the difficulty in staining embryonic tissue by conventional histological techniques, as already pointed out by Ramón y Cajal (1899). Many aspects of the behavior and function of dendritic filopodia remain a mystery. For example, controversy exists as to whether or not filopodia give rise to spines. It is tempting to consider filopodia as precursors to spines because they both protrude from dendrite shafts and because their expression during development precedes that of spines. On the basis of this temporal sequence, it has been proposed that filopodia metamorphose into spines (Dailey and Smith, 1996; Ziv and Smith, 1996). However, the more elongated shape of dendritic filopodia, reminiscent of that of axonal filopodia, suggests instead an exploratory role. Given that filopodia can occur transiently in certain types of nonspiny neurons (Lund et al., 1977; Difiglia et al., 1980; Mason, 1983; Dvergsten et al., 1986; Ulfhake and Cullheim, 1988; Wong et al., 
1992; Linke et al., 1994), this probing function of filopodia seems to be unrelated to spinogenesis. Because synapses can be found on dendritic filopodia (Vaughn et al., 1974; Saito et al., 1997; Fiala et al., 1998), these protrusions might contact axons to establish early synapses, independently of the eventual formation of spines (Fiala et al., 1998). Another potential role for dendritic filopodia is in guiding the growth of dendrites (Vaughn, 1989; PorteraCailliau and Yuste, 2001), a function analogous to that of axonal growth cone filopodia. The goal of the present study was to begin to test these hypotheses by investigating the behavior of dendritic filopodia in living neurons.

We have studied the dynamics of dendritic filopodia of pyramidal neurons in acute slices using two-photon time-lapse movies with 30-sec time resolution. Our studies reveal five findings and support the notion that there exist two populations of filopodia: (1) dendritic filopodia are highly motile, in an actindependent manner, over a time scale of seconds, and behave differently than axonal filopodia; (2) dendritic protrusions are developmentally regulated: they become shorter and more densely packed, and their motility decreases as their lifetimes increase throughout the first 2 weeks of postnatal development; (3) dendritic growth cone filopodia are longer and have shorter lifetimes and greater motility than shaft filopodia; (4) dendritic shaft filopodia, but not growth cone filopodia, are regulated by neuronal activity along a continuum of dendritic protrusions; and (5) shaft filopodia, but not growth cone filopodia, elongate in response to focal glutamate application. We propose that these different populations of filopodia implement different functions in neuronal development.

\section{Materials and Methods}

Acute slices. Timed-pregnant female C57/B6J mice (Taconic Farms, Germantown, NY) or GFP-M transgenic mice (Feng et al., 2000) were kept in our animal housing facility at $12 / 12 \mathrm{hr}$ light/dark cycles at $22^{\circ} \mathrm{C}$. Pups at different ages [postnatal day (P) 0 through P12] were anesthetized by hypothermia (younger animals) or by intraperitoneal injection of ketamine-xylazine (older animals) and decapitated. The brains were quickly removed and transferred to ice-cold artificial CSF (ACSF) containing (in mM): $126 \mathrm{NaCl}, 3 \mathrm{KCl}, 3 \mathrm{MgSO}_{4}, 1.14 \mathrm{NaH}_{2} \mathrm{PO}_{4}, 1 \mathrm{CaCl}_{2}, 26$ $\mathrm{NaHCO}_{3}$, and 10 dextrose, bubbled with $95 \% \mathrm{O}_{2} / 5 \% \mathrm{CO}_{2}$ to a final pH of 7.4. Acute coronal brain slices $(300 \mu \mathrm{m})$ that included primary visual or somatosensory cortices were obtained using a vibratome (VT1000S; Leica, Bannockburn, IL). The slices were then incubated at $37^{\circ} \mathrm{C}$ for 15-30 min. Because mature neurons can have alterations in dendritic spines related to slicing, we left slices in the same ACSF at room temperature for at least $60 \mathrm{~min}$ before imaging, to allow dendrites to equilibrate after slicing (Kirov et al., 1999).

Intracellular labeling of neurons with Alexa-488. Slices were placed on a chamber maintained at $35-37^{\circ} \mathrm{C}$ on the stage of an upright Olympus BX50WI microscope (Olympus, Melville, NY). Slices were submerged in ACSF containing (in mM): $126 \mathrm{NaCl}, 3 \mathrm{KCl}, 2 \mathrm{MgSO}_{4}, 1.14 \mathrm{NaH}_{2} \mathrm{PO}_{4}, 2$ $\mathrm{CaCl}_{2}, 26 \mathrm{NaHCO}_{3}$, and 10 dextrose, perfused at a rate of $2-4 \mathrm{ml} / \mathrm{min}$, and bubbled with $95 \% \mathrm{O}_{2} / 5 \% \mathrm{CO}_{2}$. Layer 5 pyramidal neurons were identified using differential interference contrast optics with a $60 \times[0.9$ numerical aperture (NA)] water immersion objective (Olympus). Cells were patched for 1-3 min, and then the pipette was removed gently. Recordings were performed with a Dagan amplifier (BVC-700A; Dagan Instruments, Minneapolis, $\mathrm{MN}$ ), and the data were filtered at $1 \mathrm{kHz}$ and digitized at $10 \mathrm{kHz}$ with a MacAdios A/D board using Superscope (GW Instruments) run on a Power PC (Macintosh). Liquid junction potentials and series resistances were manually compensated for standard patch pipettes ( 7-16 $\mathrm{M} \Omega$ tip resistance) pulled on a Brown/Flaming microelectrode puller (P-97; Sutter Instruments, Novato, CA). Pipettes were filled with an intracellular solution containing (in mM): $5 \mathrm{NaCl}, 20 \mathrm{KCL}$, 10 HEPES, $125 \mathrm{KMeSO}_{4}$, 2.5Mg-ATP, $0.3 \mathrm{Na}-\mathrm{GTP}$, and 2 Alexa-488
(Molecular Probes, Eugene, OR). Neurons were recorded using wholecell patch-clamp technique in current clamp configuration.

Pharmacology and PicoSpritzer delivery of glutamate. Cytochalasin D $(0.5 \mu \mathrm{g} / \mathrm{ml})$, CNQX $(20 \mu \mathrm{M}), \mathrm{D}-\mathrm{APV}(40 \mu \mathrm{M})$, and TTX $(1 \mu \mathrm{M})$ were added to the perfused ACSF in selected experiments (all four chemicals were from Sigma, St. Louis, MO). Imaging was started 5-30 min after bath application of the drugs. For calcium-free experiments, ACSF was made with no added calcium, and, in its place, $2 \mathrm{mM} \mathrm{MgCl}_{2}$ was added. For washout experiments, dendrites were imaged $10-45 \mathrm{~min}$ after changing to normal ACSF. For glutamate puffing experiments, a glass electrode filled with $20 \mu \mathrm{M}$ to $1 \mathrm{~mm}$ glutamate (dissolved in ACSF and containing $0.05 \mathrm{~mm}$ Alexa- 488 to visualize the pipette while imaging) was lowered into the slice and placed within $10-50 \mu \mathrm{m}$ from the dendrite of interest. The electrode was connected to a PicoSpritzer III (Parker Hannifin Corporation, Fairfield, NJ), which delivered brief air puffs (50-200 $\mathrm{msec}$, at 5-20 psi) that propelled the glutamate onto the slice. Generally, the dendrite was imaged for 10 frames (every $60 \mathrm{sec}$ ) before the first puff of glutamate and then for an additional 20-30 frames during which two to three additional puffs were delivered.

Imaging, image processing, and analysis. Images were collected with a $60 \times(0.9 \mathrm{NA})$ water immersion objective (Olympus) using a custombuilt two-photon laser-scanning microscope (Majewska et al., 2000b; Nikolenko et al., 2003), consisting of a modified FLUOVIEW (Olympus) confocal microscope (controlled with FLUOVIEW software) and a Ti/ sapphire laser providing 720- to 850-nm 130 fs pulses at $75 \mathrm{MHz}$ (Mira; Coherent Radiation, Palo Alto, CA) pumped by a solid-state source (Verdi; Coherent Radiation). While imaging at $800 \mathrm{~nm}$, the emitted fluorescence was detected by using an internal photomultiplier tube (HC125-02; Hamamatsu, Ichinoko, Japan) in whole-area detection mode. Images of dendritic protrusions were acquired at the high digital zoom $(7 \times$ to $10 \times)$, resulting in a nominal spatial resolution of $21-30$ pixels per micrometer. For time-lapse imaging, 7 - to $10-\mu \mathrm{m}$-deep Z-stacks (usually composed of six slices, each 1.0-1.4 $\mu \mathrm{m}$ thick) were collected so that all filopodia protruding from the dendritic structure

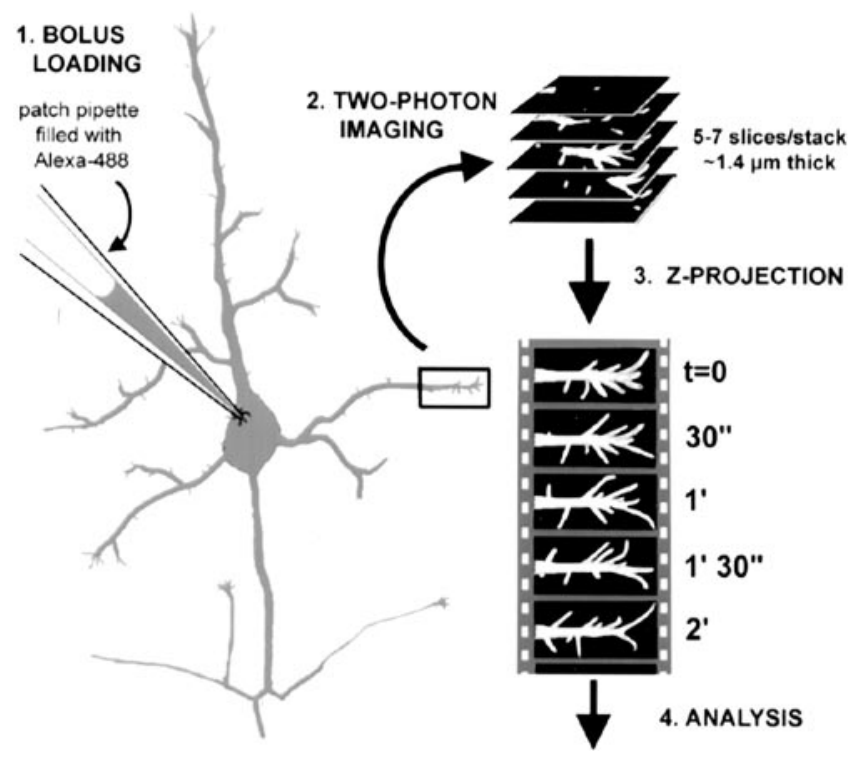

Figure 1. Bolus of neurons with Alexa-488 and two-photon imaging of filopodia. Step 1 (bolus loading): cortical pyramidal neurons in layer 5 were identified in acute slices from early postnatal mice under differential interference contrast optics and then patched with pipettes containing $2 \mathrm{~mm}$ Alexa-488. This dye diffuses quickly ( $<5 \mathrm{~min}$ ) throughout the entire cell. Step 2 (two-photon imaging): selected dendrites were imaged with a custom made two-photon microscope using $800 \mathrm{~nm}$ excitation light. Twenty stacks, each composed of 5-7 confocal slices (1-1.4 $\mu \mathrm{m}$ apart) in the XY plane, were acquired every 30 sec. Step 3 (Z-projection): using ImageJ software, the individual slices for each time point were projected along the $z$-axis into a single image. Ten-minute-long (20 time points) time-lapse movies of dendritic protrusions were, thus, generated. Step 4 (analysis): the lengths and density of dendritic protrusions were measured using the ROI tool in ImageJ (see Materials and Methods and Fig. 3). 


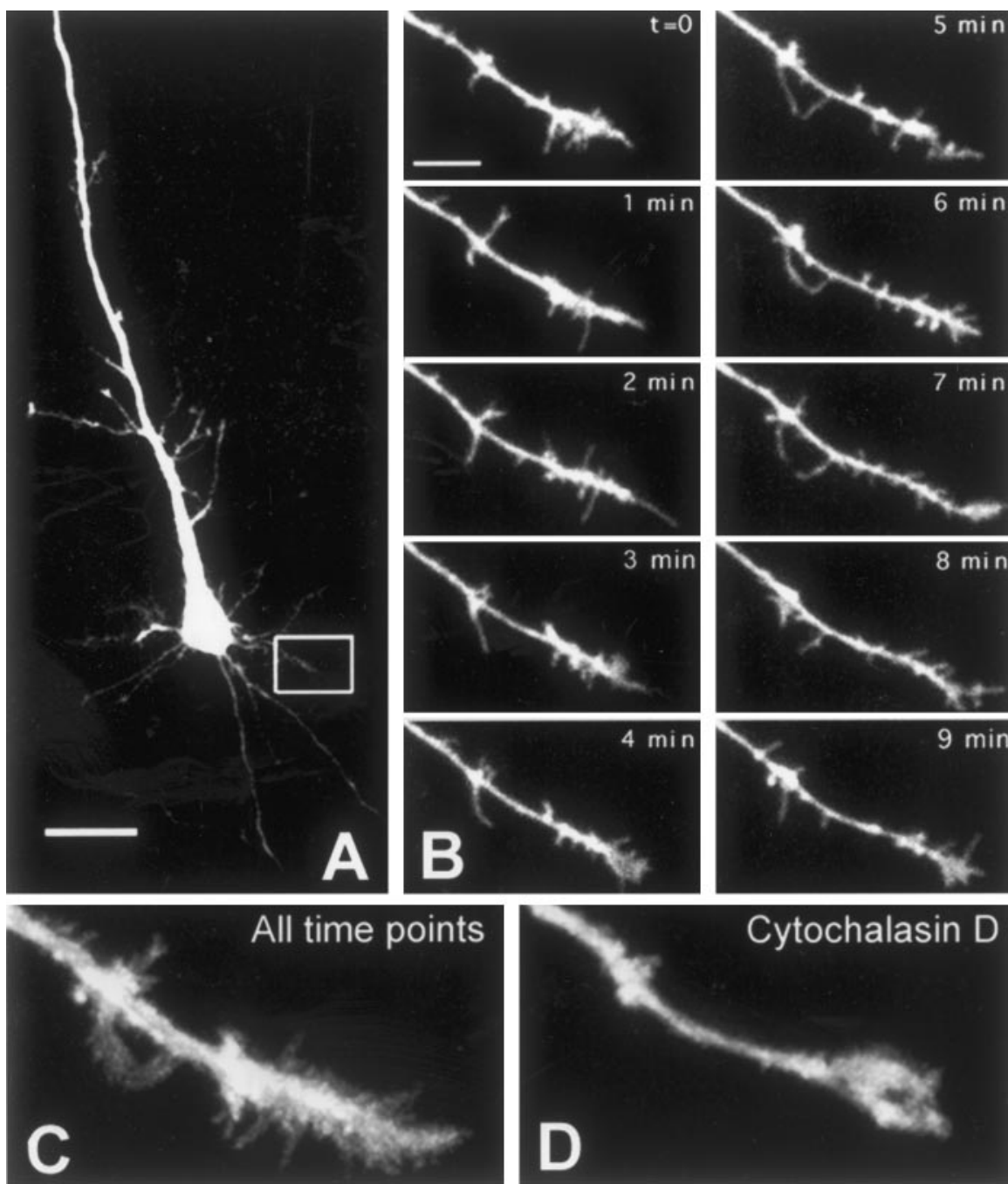

Figure 2. High-resolution two-photon imaging of dendritic filopodia in early postnatal neurons reveals their dynamism: motility is actin dependent. $A$, Layer 5 pyramidal neuron in visual cortex of a P2 mouse imaged by two-photon microscopy. Inset, Dendrite imaged in B. Scale bar, $25 \mu \mathrm{m}$. B. Time-lapse movie of dendrite from cell shown in $A$ (see movie 1 in supplementary data, available at www.jneurosci.org). Numerous filopodia protrude in and out of the dendrite. Note that filopodia seem to be clustered at the dendrite tip (growth cone) compared with the proximal shaft (see also Figs. 6 and 7). Images were acquired every 30 sec, but only half the time points are shown here. Scale bar, $5 \mu \mathrm{m}$. C, Collapsed view of all frames of the movies shown above. This displays nicely the exploratory behavior of filopodia throughout the 10-min imaging period. D, Collapsed view of all 20 frames from a 10 -min movie of the same dendrite, 5 min after applying cytochalasin $\mathrm{D}(1 \mu \mathrm{g} / \mathrm{ml})$, an actin polymerization inhibitor, into the bath (see movie 2 in supplementary data, available at www.jneurosci.org). Cytochalasin D significantly reduced filopodia motility (see Fig. 9A).

were imaged. Stacks were collected every $30 \mathrm{sec}$. For each time point, five to eight focal planes were projected into a single image (Fig. 1). The lengths of all protrusions in every movie were obtained by manually drawing skeleton versions of the dendrites with their protrusions (Fig. 3). The analysis was done blindly on unprocessed images, and there was no inter-rater variability between two observers (C.P-C. and D.T.P.). Filtering was used subsequently only for display purposes in the final figures shown here. All image processing and analysis were done with customwritten macros in ImageJ (http://rsb.info.nih.gov/ij/). Images were aligned manually to correct for drift in the XY planes. Movies containing frames that drifted out of focus ( $z$-axis) were discarded. Motility was calculated as the absolute difference in length of protrusions from frameto-frame, divided by the total number of frames. The value is, thus, expressed in micrometers per $30 \mathrm{sec}$. Lifetime (in minutes) was calculated as the number of frames in which a particular protrusion was visible, divided by two. All statistical analyses were performed with Excel software (Microsoft, Redmond, WA) using Student's $t$ tests. Error bars in graphs represent the SEM. In the text and in figure legends, $n$ indicates the number of protrusions and $N$ the number of dendrite segments analyzed (or the number of experiments for pharmacological manipulations).

Our length and motility measurements for filopodia are likely to be slight underestimates of the actual values because the $z$-axis projection partially obscures the true lengths of filopodia going in and out of the final Z-plane. Motility measurements would be further underestimated at early ages by the fact that we did not take into account motility derived from the bending, branching, or sweeping behavior of filopodia (Fig. 4).

\section{Results}

Two-photon imaging reveals different types of motility of dendritic filopodia Layer 5 cortical neurons were imaged with two-photon microscopy in acute slices after intracellular labeling with the fluorescent dye Alexa-488 (Fig. 1) (see Materials and Methods). This bolus-loading method (Majewska et al., 2000a) permitted excellent visualization and resolution of dendritic filopodia as early as $\mathrm{P} 0$, without delays because of gene expression, as occurs with viral-mediated or gene-gun-based biolistic green fluorescent protein (GFP) transfection systems (Lo et al., 1994; Maletic-Savatic et al., 1999). In time-lapse movies with images acquired at $30-\mathrm{sec}$ intervals, filopodia protruded in and out of dendritic shafts with remarkable dynamism (see movie 1 in supplementary data, available at www.jneurosci.org). At early postnatal ages (P2-P3), filopodia were short-lived, with the vast majority of filopodia appearing and disappearing within the 10-min imaging period (Figs. 2 and 3 ).

To quantify the filopodia motility, skeleton drawings of dendritic protrusions at every frame of the 10-min movies were obtained (Fig. 3). Measurements of the lengths and numbers of protrusions in each frame of these movies allowed us to obtain values for the average lengths and motility (average length change from frame to frame) as well as for the average density and lifetime (i.e., the duration of the movie in which an individual protrusion could be detected) of protrusions. The maximum velocity of individual filopodial extension was $\sim 3 \mu \mathrm{m} / 30 \mathrm{sec}$. The formidable range of motile behavior included extension and retraction, bending, branching, and even rotation (sweeping) along the dendritic shaft (Fig. 4). The vast majority of filopodia simply extended and retracted without branching, but sweeping and bending movements were also rather frequent at early ages $(<\mathrm{P} 6)$. However, during the second week of postnatal development, protrusions simply extended and retracted, without branching, sweeping, or bending.

We often observed hotspots of high filopodia activity in dendritic shafts at early postnatal ages (P2-P5). Thus, several filopodia usually protruded from specific locations along the dendritic shaft over the 10-min imaging period (see Fig. 6, white arrows). These hotspots occurred at irregular intervals (usually $\sim 5-10$ 
$\mu \mathrm{m}$ apart), and in between these nodes of high filopodia activity were relatively quiet segments of dendrite.

One of the advantages of two-photon microscopy is the reduced risk of photo damage compared with conventional confocal microscopy (Denk and Svoboda, 1997). Nevertheless, prolonged exposure of living tissue to mode-locked infrared light, as occurs in our time-lapse recordings, can still lead to photo damage manifested as arrest of protrusion motility, resorption of filopodia, and eventually blebbing of membranes into a rosary-like beaded pattern. Under our imaging protocols, we did not observe photobleaching or phototoxicity (as described previously) with average laser power under $4 \mathrm{~mW}$ at the specimen. Dendrites that did not exhibit motility or were devoid of filopodia were easily detected and excluded from the analysis. To further control for photo damage, we compared the first 10 time points and the last 10 time points of movies at P2-P5 ( $N=23$ dendrite segments) and found no statistically significant differences in length or density of filopodia between the beginning and the end of the imaging period ( $p=0.37$ and 0.92 , respectively) (data not shown). This suggests that photo damage was not a confounding factor in our experiments. Furthermore, we have imaged dendrites for more extended periods of time (60-min movies), and, qualitatively, we found no obvious change in filopodia.

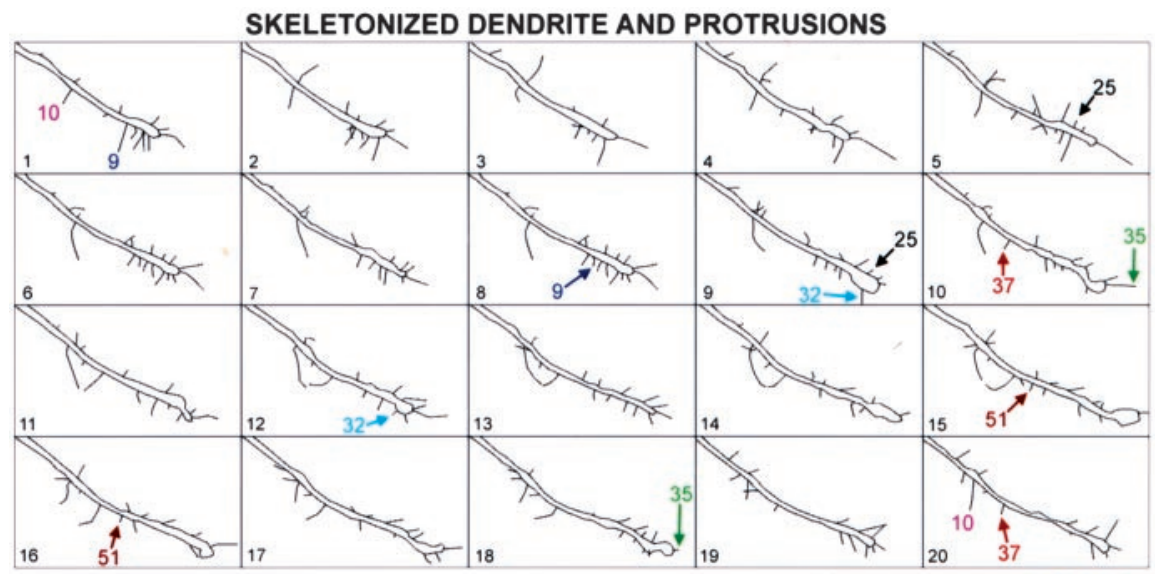

\section{FILOPODIA LENGTHS OVER TIME}

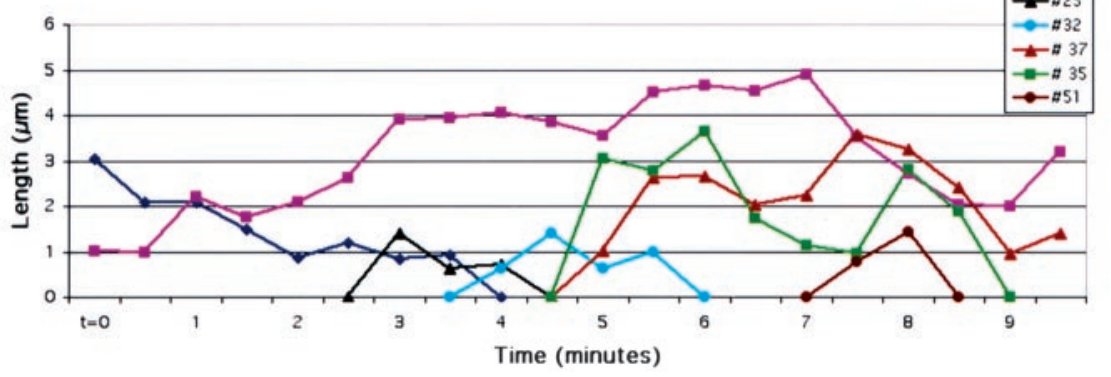

Figure 3. Dendritic filopodia are highly motile protrusions in early postnatal development. Top, Skeleton representation of all 20 frames of the movie shown in Figure 2. Individual protrusions were traced in ImageJ (see Materials and Methods) in all 20 frames of each time-lapse movie. Skeleton versions of movies of filopodia and other protrusions were drawn for all dendrites imaged in this study throughout development from P2 through P12 (a total of 56 dendrite segments and 1008 filopodia), to measure their lengths using custom-written macros in ImageJ. A few representative filopodia are labeled; arrows point to the first and last frame in which those filopodia can be distinguished. Bottom, Graph displaying the lengths of the representative filopodia mentioned above over the same 10-min movie. Most filopodia appear and disappear over the 10-min imaging period (e.g., filopodia \#25, \#32, and \#51). At these early ages, only rare filopodia were present throughout the length of the movie (e.g., filopodium \#10).

\section{Filopodial motility is actin based and is also present in GFP-expressing neurons}

Because motility of dendritic spines is actin based (Fischer et al., 1998), we wondered whether filopodia motility was similarly regulated by the actin cytoskeleton (Figs. $2 \mathrm{D}$ and $10 \mathrm{~A}$ ) (see movie 2 in supplementary data, available at www.jneurosci.org). We tested this by bath application of cytochalasin $\mathrm{D}(N=4$ experiments; $n=192$ filopodia), an inhibitor of actin polymerization. In the presence of cyctochalasin $\mathrm{D}$, we observed an $\sim 45 \%$ decrease in the density of filopodia $(p=0.004)$, an $\sim 55 \%$ decrease in the average filopodia motility $(p=0.011$ ), and an $\sim 75 \%$ increase in the average lifetime of filopodia from 4.2 to $6.5 \mathrm{~min}$ ( $p=0.016$ ). An $\sim 15 \%$ decrease in the average length of filopodia and an $\sim 70 \%$ decrease in turnover did not quite reach significance ( $p=0.074$ and $p=0.083$, respectively). The changes in density, lifetime, and turnover all recovered after washing out the drug $(p=0.54, p=0.43$ and $p=0.94$, respectively, compared with control; $N=3$ experiments with $n=90$ additional filopodia), but this recovery required several hours ( $\geq 4 \mathrm{hr}$ ).

We also investigated whether the bolus injection could affect the dynamic behavior of filopodia, because either the trauma of patching, the cytosolic dialysis associated with whole-cell recordings, or our choice of dye could potentially alter the motility. Thus, as a control, we also imaged dendritic shaft protrusions of GFP-expressing neurons in retrosplenial and cingulate cortex from GFP-M transgenic mice (Feng et al., 2000). At P8-P12, we

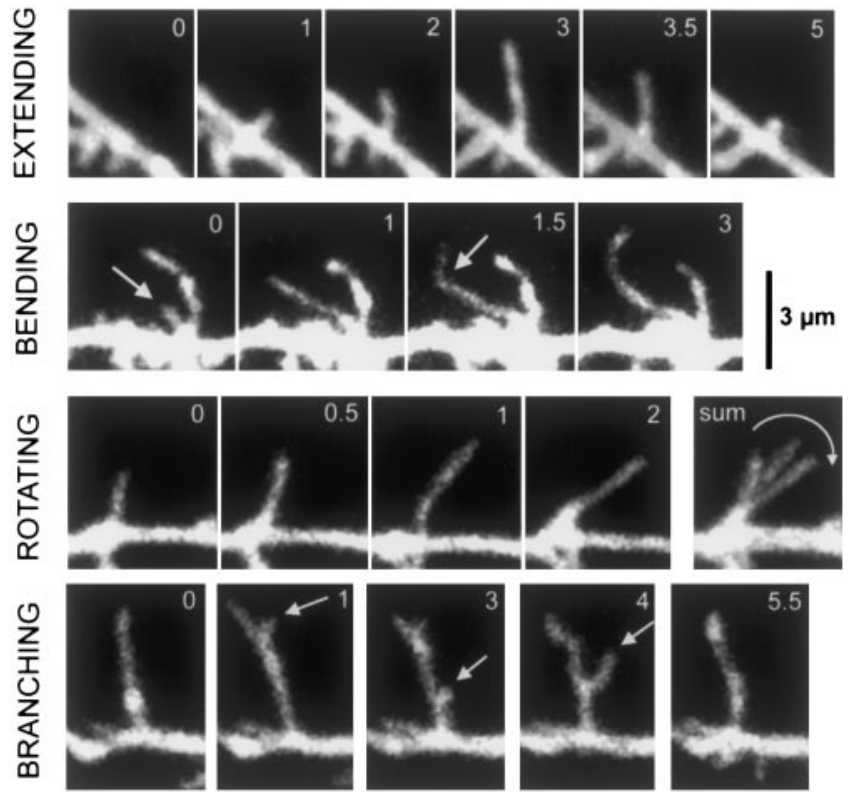

Figure 4. Dendritic filopodia exhibit a wide range of motile behaviors. The motility of dendritic filopodia at P2-P5 was manifested by a wide range of behaviors. This included extension and retraction (first row), bending along the stem of the filopodium (second row), rotation around an axis at the base of the filopodium (third row), or branching (fourth row). Scale bar, 3 $\mu \mathrm{m}$ (for all panels). 


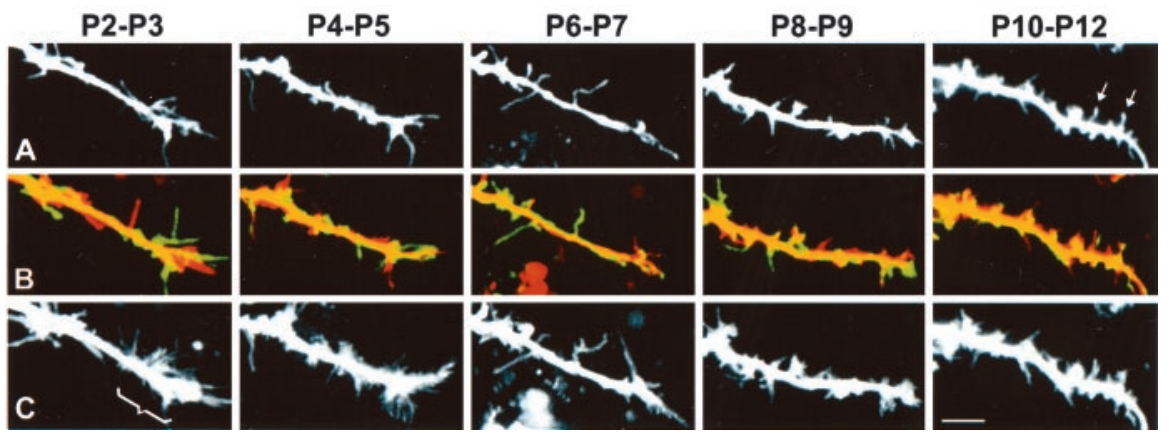

Figure 5. Dendritic filopodia are developmentally regulated; dendritic growth cones disappear after P5. The first row $(A)$ shows representative dendritic segments of cortical neurons of mice at P2 through P10 (see movies 3-7 in supplementary data, available at www.jneurosci.org). The second row $(B)$ shows composite views of the first (red) and last (green) frames of the 10-min time-lapse movies of the dendrites. The third row ( $($ ) shows a collapsed view of all 20 frames of the 10-min movie at each postnatal age. Scale bar, $5 \mu \mathrm{m}$. These panels show four results. First, filopodia are longer and more densely packed in dendritic growth cones than in shafts. Note that dendritic growth cones (bracket in C) are only apparent at P2-P3 and P4-P5. Second, dendritic protrusions become progressively more densely packed throughout early postnatal development and become shorter in dendrite tips. Third, as judged by the overlap between first and last time frames in each movie (second row), a switch from highly motile, transient filopodia to more stable, immobile protrusions occurs throughout early postnatal development. Fourth, spinelike protrusions, with bulbous swellings at their tips (heads), begin to appear at P10 -P12 (arrows in A).

found no appreciable differences between control Alexa-labeled neurons and GFP-expressing neurons $(N=6 ; n=157)$ in the motility ( 0.35 vs $0.32 \mu \mathrm{m} / 30 \mathrm{sec} ; p=0.56)$, density ( 0.6 vs 0.7 protrusions $/ \mu \mathrm{m} ; p=0.1$ ), or lengths ( 1.2 vs $1.5 \mu \mathrm{m} ; p=0.09$ ) of dendritic protrusions, suggesting that the bolus-loading technique was innocuous to neurons.

\section{Developmental regulation of dendritic filopodia}

We next examined the developmental time course of the expression of early dendritic protrusions. We prepared acute slices from mice at different postnatal ages from P0 to P12 and imaged dendrites of layer 5 pyramidal neurons in primary visual and somatosensory cortices, at 30-sec intervals. A total of 56 dendrite segments and 1008 protrusions in growth cones and shafts were analyzed in 10-min movies. Approximately five stages of protrusion development were identified (Fig. 5) (see movies 3-7 in supplementary data, available at www.jneurosci.org). At P0-P1, pyramidal neurons had simplified dendritic trees relatively devoid of protrusions, except for scattered growth cone filopodia at the tips of a few growing dendrites (data not shown). These young neurons had an apical dendrite without branches and usually fewer than two or three shorter basal dendrites. In contrast, the growing axon had prominent growth cone filopodia that were highly motile. At P2-P5, the dendrites had many filopodia at their tips (forming typical dendritic growth cones) but somewhat fewer filopodia in the shafts. Filopodia were very dynamic and were particularly active and long at the dendrite tips (Figs. 2 and 6). By P6-P7, the neurons had more mature dendritic trees, and most dendritic tips lacked the typical growth cones seen at earlier ages. Instead, filopodia seemed evenly distributed throughout the dendritic tree. By $\mathrm{P} 8-\mathrm{P} 9$, protrusions were shorter and more stable but were still devoid of any bulbous swellings (spine heads) at their tips. By P10-P12, most protrusions were stable throughout the 10-min time-lapse movies, and some resembled spines because they had developed typical heads. Nevertheless, even at these older ages, a small number of filopodial protrusions still appeared and disappeared quickly within the 10-min imaging period.

In summary, we observed several distinct patterns of protrusion expression: (1) there was a switch from very transient and highly motile protrusions in the first few days of postnatal life to more stable and less motile protrusions in the second week of development; and (2) there was a corresponding morphological change from filopodia-like protrusions (long, thin) in the first week of postnatal life to spine-like protrusions (short, with heads) in the second week of life. Subsequent analysis of various parameters of dendritic protrusions confirmed these observations (see below and Fig. 7).

\section{Differences among filopodia in} dendritic growth cones and shafts, and axonal growth cones

We next compared the physical characteristics and dynamic behavior of filopodia in dendritic shafts, dendritic growth cones, and axonal growth cones (Figs. 5 and 6). First, we compared dendritic filopodia in shafts versus growth cones. Filopodia in dendritic growth cones are more densely packed and were longer than those in dendritic shafts (Fig. 6, compare left and middle columns; Fig. 7). Next, we compared filopodia in dendritic versus axonal growth cones (Fig. 6, compare middle and right columns). Axonal growth cones had a more fanned morphology, with filopodia protruding at acute angles from the tip of the dendrite (Fig. 6, right column). Interestingly, the typical webbed appearance that is traditionally associated with axonal growth cones in cultured neurons was also observed in axonal growth cones in acute slices (Fig. 6, right column), but not in dendritic growth cones (Fig. 6, middle column). Moreover, axonal filopodia did not bend as frequently and almost never had the branching behavior that was observed in dendritic filopodia (Fig. 4). Quantitative analysis revealed that axonal filopodia ( $n=36$, from three axon growth cones) were, on average, $50 \%$ longer (3.3 vs $2.2 \mu \mathrm{m} ; p<0.001$ ) (data not shown) and $50 \%$ more densely packed ( 2.1 vs 1.4 protrusions/ $\mu \mathrm{m} ; p<$ 0.001 ) (data not shown) than their dendritic counterparts at the same age.

\section{Evidence for two types of filopodia in dendritic shafts and growth cones at P2-P5}

We sought to establish whether the qualitative differences observed in filopodia from dendritic shafts and growth cones were indeed significant (Figs. 6 and 7). To distinguish filopodia in dendritic tips versus shafts, we divided terminal dendrites into two regions, such that the distal $5 \mu \mathrm{m}$ segment was considered as the tip and the remaining proximal part was labeled "shaft". This division point was based on a rough estimate of the length of typical growth cones by visual inspection. Moreover, using a cutoff of $>5 \mu \mathrm{m}$ did not capture as great a difference between growth cone and shaft filopodia. It should be noted that the actual boundary between shaft and growth cone might actually vary slightly from dendrite to dendrite. In the shaft category, we included both dendrite segments proximal to the tips but also additional shaft segments chosen at random.

When comparing dendritic tips $(n=129 ; N=7)$ to shafts $(n=163 ; N=8)$ at P2-P3, several statistically significant differences were found. First, the density of filopodia was approximately three times greater in growth cones than in shafts (1.31 vs 0.45 filopodia per micrometer; $p<0.001$; Fig. 7A). Second, 


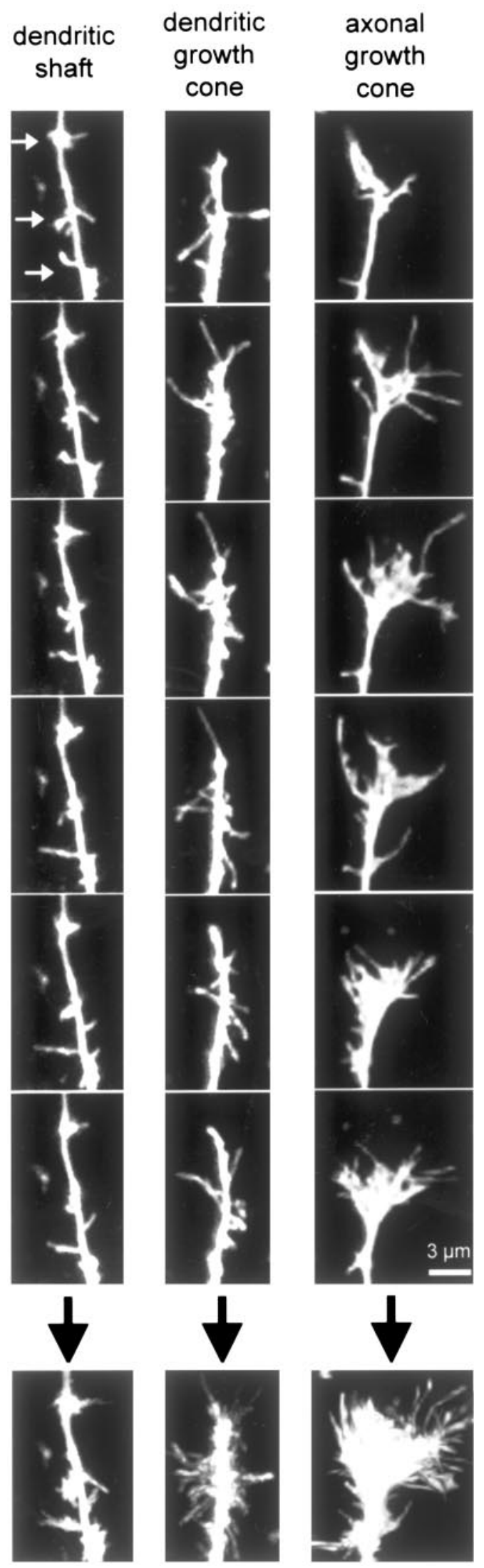

Figure 6. Differences between filopodia in axonal and dendritic growth cones and between filopodia in dendritic growth cones and in dendritic shafts. Left, Example of a typical dendritic shaft at P2-P5. Note that filopodia are shorter than in both axonal growth cones and dendritic growth cones, and they are less densely packed. There are also three hotspots of filopodia activity (white arrows). These hotspots were frequently seen in young dendrites but almost growth cone filopodia were, on average, $\sim 37 \%$ longer than shaft filopodia (2.18 vs $1.59 \mu \mathrm{m} ; p=0.019$; Fig. $7 B)$. Third, motility was $\sim 25 \%$ greater for growth cone filopodia than for shaft filopodia ( 1.72 vs $1.38 \mu \mathrm{m} / \mathrm{min} ; p=0.0011$; Fig. $7 C$.). Despite this greater motility, there was no difference in the lifetime of filopodia between dendritic growth cones and shafts at that early age (both $3.1 \mathrm{~min} ; p=0.87$; Fig. 7D). Taking into account that growth cone filopodia at early ages are able to fill the entire neuropil surrounding the growth cone in $<10 \mathrm{~min}$ (Fig. $2 \mathrm{C}$ ), we estimate that filopodia in a typical $5 \mu \mathrm{m}$ growth cone at P2-P3 (average maximum length of filopodia, $2.5 \mu \mathrm{m}$ ) can explore an $\sim 50 \mu \mathrm{m}^{3}$ volume of neuropil.

As a separate index, we also calculated a protrusion turnover rate, defined as the average number of protrusions that appeared or disappeared every minute, per $10 \mu \mathrm{m}$ segment of dendrite. We found that, at $\mathrm{P} 2-\mathrm{P} 3$, the number of filopodia lost or gained every minute was more than three times greater in growth cones than in shafts (3.8 vs 1.2 filopodia/min per $10 \mu \mathrm{m}$ segment of dendrite; $p=0.011$; Fig. $7 E)$.

\section{Dendritic growth cones disappear after P5}

Smaller differences in density, length, and motility of protrusions located on dendritic tips versus shafts persisted at P4-P5 (Fig. 7; $p<0.05$ for all three comparisons), suggesting that dynamic growth cones still existed at the tips of dendrites at that age. Interestingly, such differences could no longer be detected at P6-P7 $(n=106 ; N=11 ; p=0.06-0.7), \mathrm{P} 8-\mathrm{P} 9(n=184 ; N=$ $10 ; p=0.11-0.52)$, or $\mathrm{P} 10-\mathrm{P} 12(n=191 ; N=13 ; p=0.1-0.52)$, and this coincided with our inability to discern dendritic growth cones after P5 (Fig. 5). At P10-P12, the average length of tip protrusions seemed shorter than that of shaft protrusions, but this difference was not statistically significant (0.93 vs $1.45 \mu \mathrm{m}$; $n=13$ and 178, respectively; $p=0.1$; Fig. $7 B$ ). However, the protrusion turnover value was now lower in tips than in shafts ( 0.09 vs 0.55 filopodia/min per $10 \mu \mathrm{m}$ segment of dendrite; $p=$ 0.002; Fig. 7E). This difference in turnover, combined with the observation that at $\mathrm{P} 10-\mathrm{P} 12$ lifetime was higher in tips than in shafts ( 8.5 vs $6.6 \mathrm{~min} ; p=0.018$; Fig. $7 D)$, suggests that tip protrusions may eventually become the most stable of all.

Finally, we also compared protrusions from apical versus basal dendrites $(n=421)$, as well as from visual versus somatosensory cortices $(n=329)$, at different postnatal ages. We found no statistically significant differences in any of the four parameters of density, length, motility, and lifetime (data not shown) ( $p>0.40$ for all comparisons, except $p=0.07$ for density of protrusions at $\mathrm{P} 10-0 \mathrm{P} 12$, which was 0.9 per micrometer in apical dendrites versus. 0.6 per micrometer in basal dendrites). This suggests that the phenomena we are observing are universal among protrusions from all cortical pyramidal neurons and from different dendritic branches within a neuron.

Dendritic protrusions become shorter, less motile, and more stable throughout development

When we quantified the density, length, motility, lifetime, and protrusion turnover throughout development, we found several

$\leftarrow$

never after P6. Middle, Example of a typical dendritic growth cone at P2-P5. Note how individual filopodia are easily identified (no webbing) and that filopodia aim in all directions, including away from the tip of the dendrite. Right, Example of a typical axonal growth cone at P2-P5. Note the long filopodia and the webbing between filopodia. Almost all filopodia are oriented at acute angles toward the tip of the axon. The different rows show different time points in the 10-min time-lapse movies. The last row (below the black arrows) shows collapsed sums of all 20 individual time points from each 10-min movie. Scale bar, $3 \mu \mathrm{m}$. 

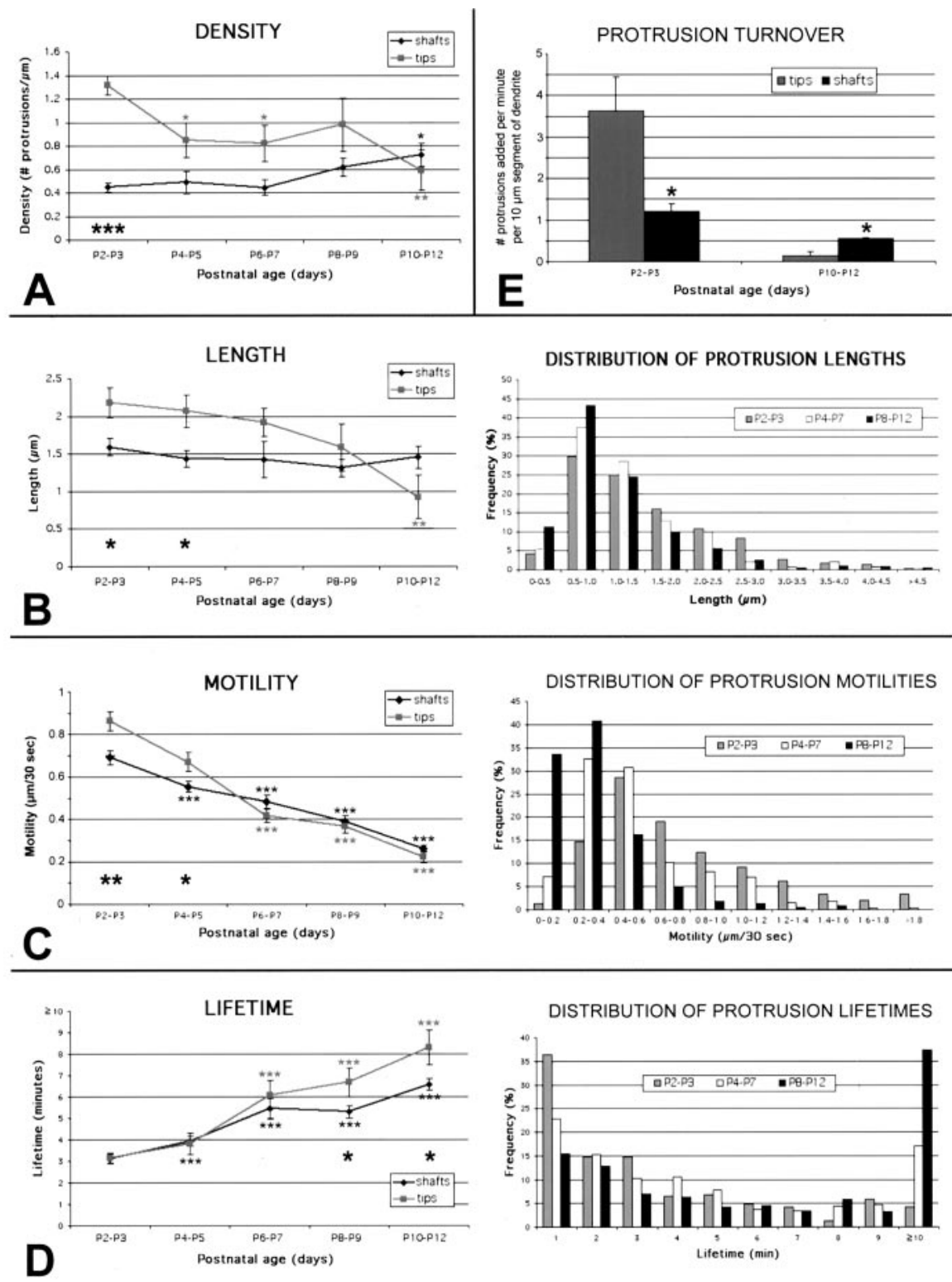

Figure 7. Developmental time course of dendritic protrusions: evidence for distinct filopodia in growth cones and shafts. A, Density. The average density of protrusions in dendritic tips decreases steadily throughout postnatal development (from 1.3 per micrometer at P2-P3 to 0.6 per micrometer at P10-P12), whereas the density of protrusions on dendritic shafts increases during the second week of postnatal development (from 0.4 per micrometer to 0.7 per micrometer). The higher density of filopodia in growth cones (as compared with those in shafts) at P2-P3 was statistically significant (large asterisks). Note that values for mice aged P8 -P12 consist of pooled data from GFP- and Alexa-labeled neurons for ages >P6, although the majority of analyzed dendrites came from Alexa-loaded neurons. $B$, Length. The average length of all protrusions on dendritic tips at any given time decreases throughout early development, as growth cones disappear (from $2.2 \mu \mathrm{m}$ at P2-P3 to $0.95 \mu \mathrm{m}$ at P10 -P12). The average length of shaft protrusions remains relatively stable at $1.5 \mu \mathrm{m}$. The longer lengths of filopodia in growth cones (as compared with those in shafts) at P2-P3 and at P4-P5 were statistically significant (black asterisks). The right panel shows the frequency distribution of the average lengths individual protrusions throughout development. $C$, Motility. The average motility of protrusions in both dendritic tips and shafts decreases throughout early postnatal development (from $\sim 0.8 \mu \mathrm{m} / 30$ sec at P2-P3 to $0.25 \mu \mathrm{m} / 30$ sec at P10 -P12). The fastest recorded speeds exceeded $3 \mu \mathrm{m} / 30$ sec. Motility of protrusions was also significantly higher in dendritic growth cones than in shafts at P2-P3 and at P4 -P5, which is when growth cones can be observed (black asterisks). The right panel shows the frequency distribution of protrusion motility throughout development. Note that the frequency of highly motile protrusions ( $\geq 0.8$ $\mu \mathrm{m} / 30 \mathrm{sec}$ ) decreases from $36 \%$ at P2-P3 to $5 \%$ at P10 -P12.D, Lifetime. The average lifetime of protrusions in both dendritic tips and shafts increases steadily throughout early development (from $3 \mathrm{~min}$ at P2-P3 to $\sim 7 \mathrm{~min}$ at P10 -P12). Lifetimes were also significantly higher in dendritic tips at P8 -P9 and at P10 -P12 (black asterisks). The right panel shows the frequency distribution of protrusion lifetimes throughout development. Note that two populations of protrusions can be distinguished on the basis of their lifetimes, one with lifetimes $\geq 8$ min (stable; i.e., protospines) and the other with protrusion lifetimes $\geq 3 \mathrm{~min}$ (transient; i.e., filopodia). For instance, $75 \%$ of all protrusions at any age fall into either of these categories. $E$, Turnover. The average turnover of dendritic tip protrusions (i.e., the number of protrusions added per minute per $10 \mu \mathrm{m}$ segment of dendrite) decreases from 3.5 at P2-P3 to 0.1 at P10 - P12. A proportionally smaller but also statistically significant decrease was observed in the turnover of shaft protrusions, from 1.2 at P2-P3 to 0.5 at P10-P12. The difference in turnover between shafts and tips was also statistically significant at P2-P3, again demonstrating the particularly high dynamism of dendritic growth cones. The reverse was true at P10-P12, indicating that tip protrusions at older ages are the most stable of all protrusions. Number of dendrites and individual protrusions analyzed for all panels: for dendritic tips at P2-P3, P4-P5, P6 -P7, P8 -P9, and P10 - P12, N = 7, 4, 3, 4, and 5, respectively. For dendritic shafts at P2-P3, P4-P5, P6 -P7, P8 -P9, and P10-P12, $N=8,7,4,6$, and 8, respectively, where $N=$ number of dendrites analyzed; for each dendrite, values are averages of 20 time frames, 30 sec apart. In terms of the number of individual protrusions $(n)$, this corresponds to: for dendritic tips at P2-P3, P4 -P5, P6 - P7, P8 -P9, and P10 - P12, $n=147,46,29,28$, and 13 , respectively; for dendritic shafts at P2-P3, P4 -P5, P6 -P7, P8 -P9, and P10-P12, $n=150,184,77,156,178$, respectively. That is a total of 1008 protrusions and a total of 56 dendrite segments. Error bars represent the SEM, which was calculated using the number of dendrites $(N)$ for measurements of density and length and using the number of protrusions $(n)$ for measurements of motility and lifetime. ${ }^{*} p<0.05 ;{ }^{* *} p<0.01 ;{ }^{* * *} p<0.001$. 
statistically significant patterns. First, the density of dendritic shaft protrusions increased by $60 \%$ from P2-P3 to P10-P12 (from 0.45 to 0.72 protrusions per micrometer; $p=0.022$; Fig. $7 A$ ). Instead, the density of dendritic growth cones/tip protrusions decreased over the same time period (from 1.31 to 0.59 protrusions per micrometer; $p=002$; Fig. $7 A$ ), again coinciding with the disappearance of growth cones from the distal most aspect of dendrites. Second, whereas the average length of dendritic tip protrusions decreased steadily throughout early cortical development (from $2.2 \mu \mathrm{m}$ at $\mathrm{P} 2-\mathrm{P} 3$ to $0.9 \mu \mathrm{m}$ at P10-P12; $p=$ 004; Fig. $7 B$ ), the slight decrease in length of shaft protrusions was not significant $(p=0.47$ ). The average length of individual protrusions throughout their lifetime followed identical trends (data not shown). The average maximum length achieved by individual shaft protrusions also decreased significantly throughout early postnatal development. For example, the average maximum length of shaft filopodia decreased from $2.0 \mu \mathrm{m}$ at P2-P3 to 1.5 $\mu \mathrm{m}$ at $\mathrm{P} 10-\mathrm{P} 12(p<0.001)$ (data not shown). Third, the motility of dendritic protrusions decreased steadily in both tips and shafts between P2-P3 and P10-P12 $(\sim 0.8 \mu \mathrm{m} / 30 \mathrm{sec}$ vs $\sim 0.2$ $\mu \mathrm{m} / 30 \mathrm{sec} ; p<0.001$; Fig. $7 C)$. Fourth, the lifetime of dendritic protrusions increased throughout the first 2 weeks of postnatal development (from 3 min at P2-P3 to $\sim 7.5$ min at P10-P12; $p<$ 0.001 ; Fig. $7 D)$. Moreover, the fraction of stable protrusions that lasted the entire 10-min movie increased from $4 \%$ at P2-P3 to $45 \%$ at P10-P12 (Fig. 7D). The distribution of protrusion lifetimes showed that $>75 \%$ of all protrusions at any age fall into either of two distinct categories of protrusions, one with lifetimes $\leq 3 \mathrm{~min}$ and another with lifetimes $\geq 8 \mathrm{~min}$ (Fig. $7 D$ ). Thus, even at $\mathrm{P} 10-\mathrm{P} 12$ when most protrusions were rather stable (persisting throughout the 10-min movie), short-lived protrusions (i.e., filopodia) were also present.

\section{Dendritic protrusions are regulated by neuronal activity and synaptic transmission}

We next investigated whether the differences between dendritic growth cones and shafts, with respect to the density, length, and motility of filopodia, had any functional significance. To do so, we tested the hypothesis that blocking neuronal activity might have differential effects on dendritic filopodia in shafts versus growth cones. It has been reported that blocking synaptic activity can lead to alterations in the density of mature dendritic spines (Kirov and Harris, 1999), but it is not clear whether filopodia are also regulated by neuronal activity. For example, in living retinal ganglion cells, the rate and extent of movement in dendritic appendages is decreased by ionotropic glutamate receptor antagonists but not by TTX, a sodium channel blocker that blocks most synaptic activity (Wong et al., 2000). Also, experiments in cultured slices have suggested that filopodia-like protrusions can emerge from dendrites after synaptic activation over a time scale of tens of minutes (Maletic-Savatic et al., 1999).

We studied the effects of activity blockade in the presence of TTX or zero-calcium conditions, to block synaptic transmission, on dendritic filopodia at P2-P5, the period of growth cones and of exclusive filopodial expression (no spines). Both of these manipulations resulted in a substantial increase in the number and length of dendritic shaft filopodia (Fig. 8A,C) (see movies 8 and 9 in supplementary data, available at www.jneurosci.org) but, surprisingly, had no effect on dendritic growth cone filopodia (Fig. $8 B$ ). On quantitative analysis, we found that, in the presence of TTX ( $N=5$ experiments), dendritic shaft filopodia $(n=216)$ were $125 \%$ longer (2.4 vs $1.7 \mu \mathrm{m} ; p=0.02)$ and $\sim 50 \%$ more densely packed ( 0.4 vs 0.6 filopodia per micrometer; $p=0.03$ )
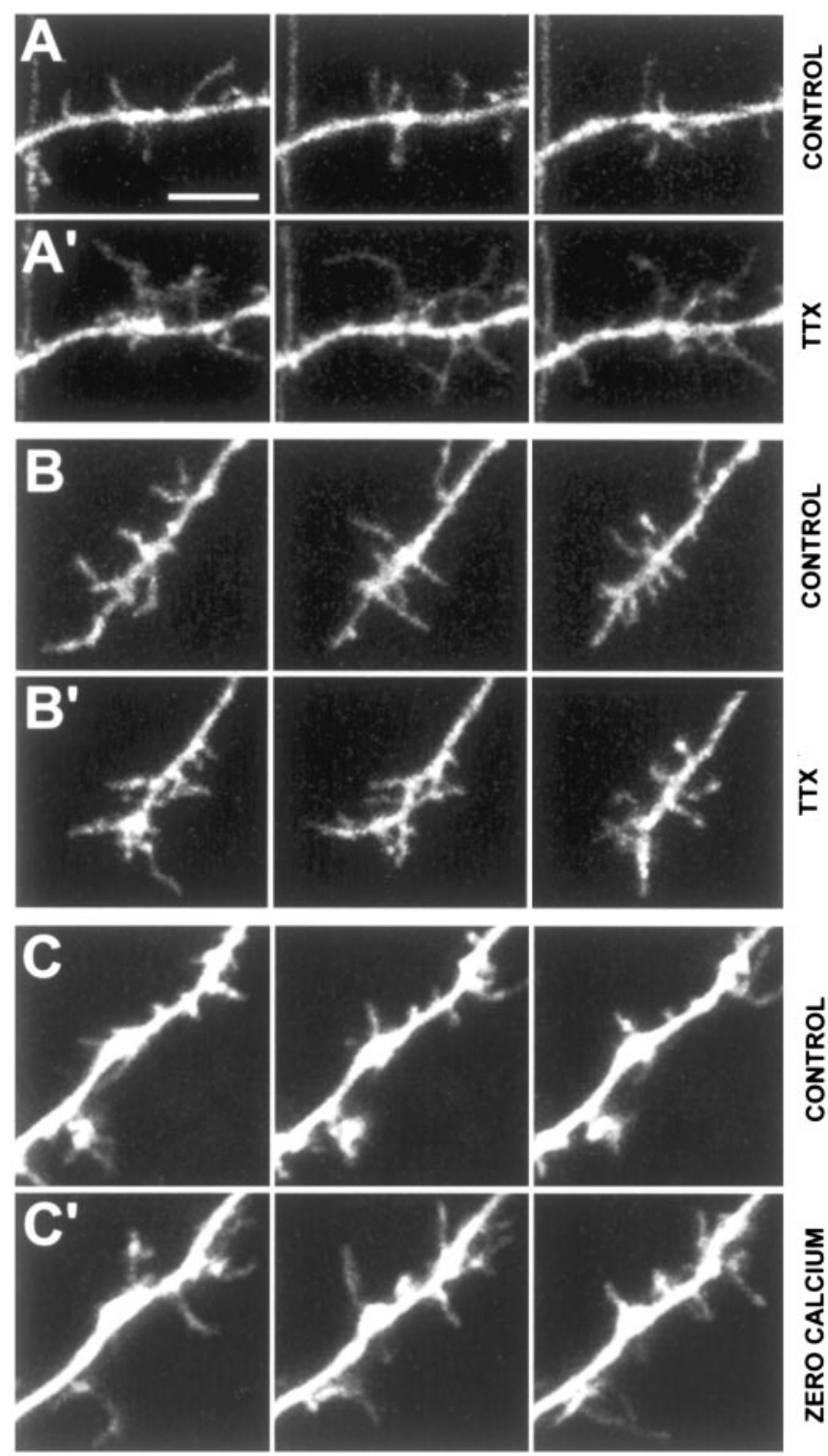

\section{$\frac{1}{2}$ (1)}

Figure 8. Blockade of global synaptic activity results in longer, more motile filopodia. $A$, Dendritic shaft from a control neuron at $\mathrm{P} 3$ in control ACSF $(2 \mathrm{~mm} \mathrm{CaCl} 2)$. The left, middle, and right panels in each row show representative snapshots of dendritic filopodia in the beginning, middle, and end of the 10 -min movies. Scale bar, $5 \mu \mathrm{m}$ (for all panels). $A^{\prime}$, Same dendritic shaft segment $\sim 7$ min after continuous bath application of $1 \mu \mathrm{m}$ TTX. Note the dramatic elongation of many filopodia in three different frames of a 10-min time-lapse movie. $B$, Dendritic growth cone from a control neuron at $\mathrm{P} 4$ in control $\left.\mathrm{ACSF}(2 \mathrm{mM} \mathrm{CaCl})_{2}\right) \cdot B^{\prime}$, Same dendritic growth cone $\sim 30$ min after continuous bath application of TTX. Note that there is no change in the density or length of growth cone filopodia. C, Dendritic growth cone from a control neuron at $P 3$ in control $\operatorname{ACSF}(2 \mathrm{~mm} \mathrm{CaCl} 2)$. See movie 8 in supplementary data, available at www.jneurosci.org. $C^{\prime}$, Same dendritic growth cone $\sim 25$ min after washing in zero-calcium ACSF. Note the dramatic elongation of many filopodia in three different frames of a 10-min time-lapse movie. This effect was reversible because filopodia lengths and motility recovered after washout. See movie 9 in supplementary data, available at www.jneurosci.org.

compared with control conditions (Fig. 10 B). Similarly, in zerocalcium ACSF, there was a $75 \%$ increase in the density ( 0.7 vs 0.4 ; $p=0.05)$ and an $\sim 45 \%$ increase in the length ( 2.0 vs $1.4 \mu \mathrm{m} ; p=$ 0.004 ) of dendritic shaft filopodia (Fig. 10C; $N=6$ experiments; $n=251$ filopodia). In contrast, we observed no effects on density or length of filopodia in dendritic growth cones using either TTX (Fig. $10 B ; N=5 ; n=177 ; p=0.7$ and $p=0.69$, respectively) or zero-calcium ACSF (Fig. $10 C ; N=4 ; n=97, p=0.52$ and $p=$ 


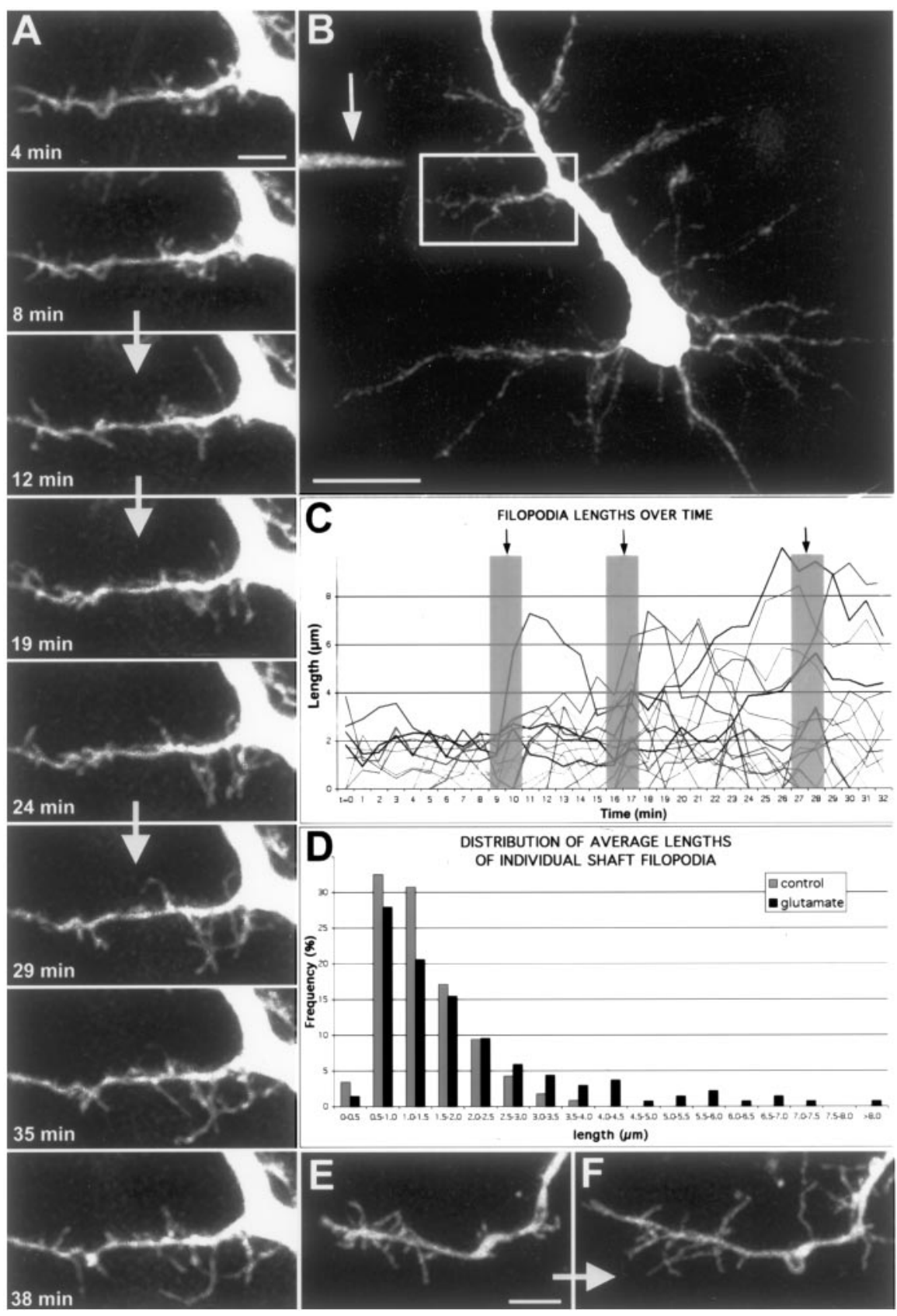

Figure 9. Focal application of glutamate results in rapid elongation of a subset of filopodia. A, Time-lapse movie of apical dendrite from a layer 5 pyramidal neuron at P4 (see movie 10 in supplementary data, available at www.jneurosci.org). Images were acquired every $60 \mathrm{sec}$, and representative time points are shown. The white arrows separating certain frames represent the times when three puffs ( $200 \mathrm{msec}, \sim 20 \mathrm{psi}$ ) of $100 \mu \mathrm{m}$ glutamate were delivered, corresponding to frames 10,17 , and 28 . Note that by the end of the movie, many filopodia have grown to lengths $>5 \mu \mathrm{m}$. Scale bar, $5 \mu \mathrm{m}$. B, In this low magnification view of a pyramidal neuron, the white box delineates the region in which the movie in $A$ was obtained. The arrow points to the location of the pipette tip containing $100 \mu \mathrm{m}$ glutamate (and Alexa-488). Scale bar, $20 \mu \mathrm{m}$. C, Graph displaying the lengths of 45 representative filopodia from the 40-min movie shown in $A$. Black arrows and gray columns designate the three times when glutamate was puffed ( $200 \mathrm{msec}$ puffs). Note that during the first 10 frames of the movie filopodia lengths never surpass $4 \mu \mathrm{m}$, whereas after just two puffs of glutamate, a subset of filopodia have reached lengths well above $4 \mu \mathrm{m}$. The effect of glutamate was quite rapid as shown by the sudden increases (within $1 \mathrm{~min}$ ) of some filopodia after individual puffs. D, Frequency distribution of filopodia lengths before and after glutamate application from five separate experiments. The gray histograms represent pooled data from 117 filopodia analyzed in the first 10 frames of each movie, whereas the black histograms represent data from 136 filopodia analyzed during 10 consecutive frames after at least two puffs of glutamate. Note that glutamate induced growth to lengths $>4 \mu \mathrm{m}$ in a minority ( $\sim 10 \%)$ of filopodia, rather than a small increase in the length of all the filopodia. $E, F$, Another example of the effects of glutamate puffing on a P2 dendrite. The pipette was located $\sim 50 \mu \mathrm{m}$ away, up and to the left of the dendrite growth cone. $E$ is a representative frame of the dendrite with a growth cone in normal conditions. $F$ is the same dendrite $\sim 30$ min after three puffs of $200 \mu \mathrm{m}$ glutamate. Scale bar, $5 \mu \mathrm{m}$.
0.88 , respectively). Interestingly, the rapid motility and lifetime of growth cone or shaft filopodia were not significantly changed by either TTX $(p>0.21$; Fig. $10 B)$ or zero calcium ACSF $(p>0.23$; Fig. $10 C$ ). The effects of zero calcium (but not those of TTX) on density and length of dendritic filopodia could be reversed $\geq 30$ min after washing in control ACSF with 2 $\mathrm{mM} \mathrm{CaCl}_{2}$ (Fig. $10 C ; N=3 ; n=44 ; p>0.4$ compared with control).

\section{Glutamate differentially regulates filopodia in dendritic shafts and growth cones}

Spiny dendrites of pyramidal cells receive predominantly excitatory glutamatergic inputs. Thus, we next asked the question of whether glutamate itself modulates dendritic filopodia at P2-P5. Blockade of NMDA and non-NMDA glutamate receptors with bath application of specific antagonists $(40 \mu \mathrm{M}$ D-APV and $20 \mu \mathrm{M}$ CNQX, respectively) led to $\sim 35 \%$ reduction in both the density $(p=0.006)$ and turnover ( $p=0.04$ ) of shaft filopodia (Fig. $10 D ; N=8 ; n=319)$ but did not affect growth cone filopodia $(p>0.56 ; N=5$; $n=164$ ). The effects of CNQX and D-APV on the density and turnover of shaft filopodia could be washed out $(p>0.63$ compared with control; Fig. $10 D ; N=4$ successful washouts; $n=116$ ). Similar effects on shaft filopodia were observed when MK-801 (another NMDA receptor antagonist) was used instead of D-APV $(N=2)$ (data not shown), but MK-801 could not be washed out.

We also tested the effects of direct application of glutamate by puffing small amounts of the neurotransmitter from a glass micropipette (connected to a PicoSpritzer), the tip of which was located $\sim 10-50 \mu \mathrm{m}$ away from the dendrite of interest. Delivery of brief puffs (50-200 msec) of 100-200 $\mu \mathrm{m}$ glutamate resulted in a sudden (within $60 \mathrm{sec}$ ) and sustained (several minutes) increase in the length of many, but not all, filopodia (Fig. 9A). In most experiments, many filopodia reached lengths of $\geq 8 \mu \mathrm{m}$, and in one case a filopodium grew toward the pipette, reaching a maximum length of $16 \mu \mathrm{m}$ (data not shown). A frequency distribution histogram of the average lengths of individual shaft filopodia demonstrates that glutamate produced a robust elongation to lengths $>4 \mu \mathrm{m}$ in only $\sim 10 \%$ of all filopodia, rather than a small increase in the length of all filopodia (Fig. 9D). This suggests that a minority of protrusions may be primed to respond to the neurotransmitter at any point in time. On aver- 
A

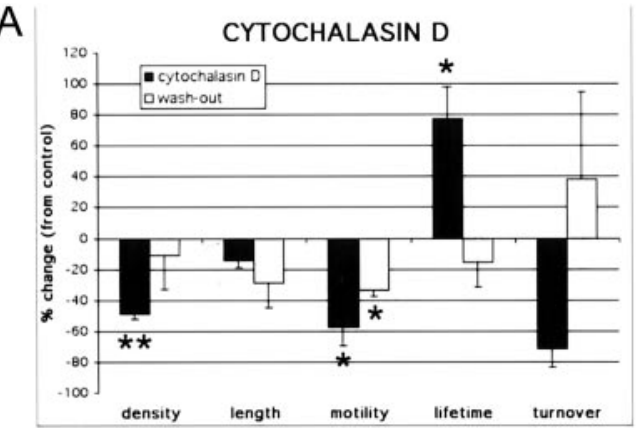

B

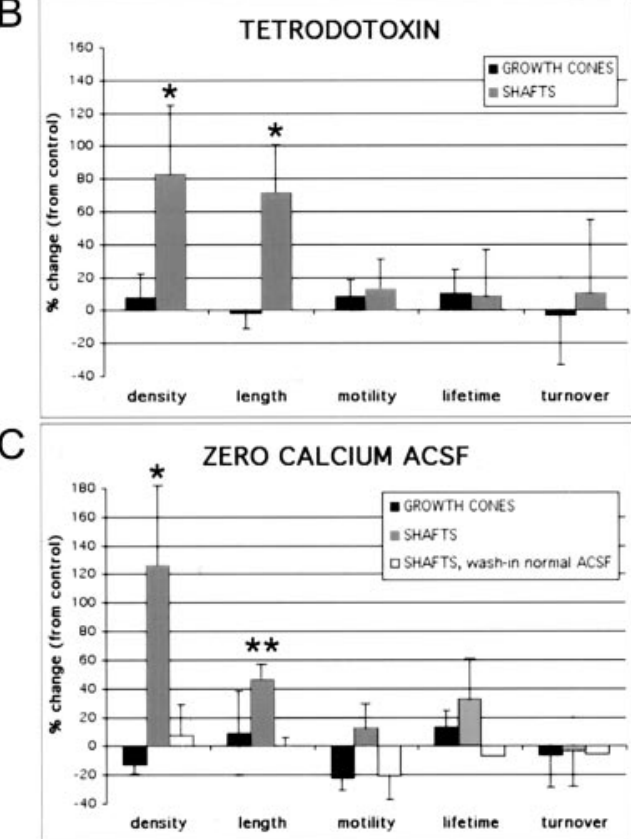

D

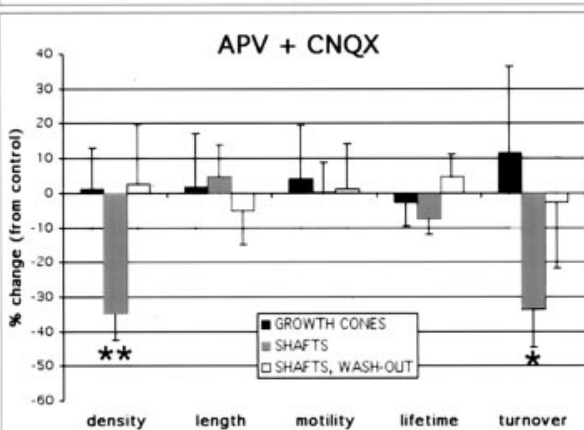

$\mathrm{E}$

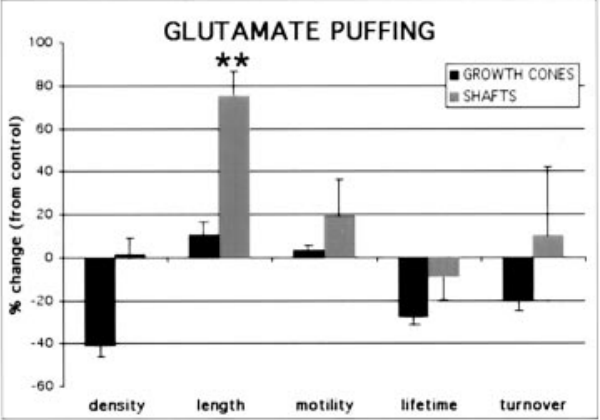

Figure 10. Dendritic shaft filopodia, but not growth cone filopodia, are regulated by neronal activity. $A$, Bath application of $1 \mu \mathrm{g} / \mathrm{ml}$ cytochalasin $D$, an inhibitor of actin polymerization ( $n=46$; four experiments), resulted in an $\sim 50 \%$ decrease in density ( $p=0.004$ ), an $\sim 55 \%$ decrease in motility ( $p=0.011$ ), and an $\sim 75 \%$ increase in lifetime ( $p=0.016$ ) of dendritic protrusions. Shaft and growth cone protrusions were equally affected. An $\sim 15 \%$ decrease in the length of filopodia approached, but did not reach significance ( $p=0.074$ ), The effect on age, glutamate led to a $75 \%$ increase in the average length of shaft filopodia $(p=0.004 ; N=5 ; n=251$; Fig. $10 E$ ) but did not significantly affect other parameters. Again, filopodia in growth cones were differentially regulated. Local application of glutamate did not result in an elongation of growth cone filopodia $(p=0.38)$, although it did cause a $40 \%$ decrease in the density ( $p=0.055$ ) of growth cone filopodia (Fig. $10 E ; N=2 ; n=76$ filopodia).

\section{Discussion}

Dendritic filopodia are nearly ubiquitously expressed in the developing CNS, yet their function is still poorly understood. Our goal was to analyze the behavior of dendritic filopodia in cortical pyramidal neurons throughout early postnatal development, to begin to understand the function of these early dendritic protrusions.

Previous studies of filopodia have used a variety of preparations, including perfusion-fixed material (Saito et al., 1997; Fiala et al., 1998), dissociated neuronal cultures (Ziv and Smith, 1996), and organotypic cultures (Dailey and Smith, 1996). Some of the in vitro studies showed that filopodia are dynamic protrusions, but the time scale used ( $\geq 6 \mathrm{~min} /$ measurement) did not allow precise measurements of their rapid motility (Dailey and Smith, 1996; Ziv and Smith, 1996). Also, because those studies relied on cultured preparations, it is not known whether the maturation of filopodia follows a similar course in the more intact CNS. Finally, none of the previous studies looked at dendritic protrusions at very early ages (e.g., P2) and were, thus, biased toward more mature, spine-like protrusions.

Building on that pioneer work, our study is a comprehensive attempt at characterizing dendritic filopodia with respect to five important parameters (density, length, motility, lifetime, and turnover) and their regulation by neuronal activity. We show that

\footnotetext{
$\leftarrow$

density and lifetime was reversible after washing out the drug, because there were no significant differences between control and washout conditions ( $p>0.26 ; n=90$; three experiments). This wash-out effect required several hours (the data come from dendrites imaged on average $\geq 4$ hr after washout of cytochalasin D). B, Blocking neuronal activity with $1 \mu \mathrm{M}$ TTX ( $n=219$; five experiments) resulted in an $\sim 85 \%$ increase in the average density of shaft filopodia ( $p=0.04$ ) and an $\sim 75 \%$ increase in the average length of dendritic shaft filopodia $(p=0.05)$. Neither the density nor the length of dendritic growth cone filopodia were significantly affected by $\operatorname{TTX}(p=0.70$ and $p=0.69$, respectively). Motility and lifetime of filopodia were unaffected by TTX in either dendritic shafts $(p>0.21)$ or growth cones $(p>0.54)$. C, Blocking neuronal activity with calcium-free ACSF ( $n=350$; six experiments) also resulted in an $\sim 125 \%$ increase in the average density ( $p=0.05$ ) and an $\sim 45 \%$ increase in the average length ( $p=0.004$ ) of filopodia in dendritic shafts. Just as with TTX, neither the density nor the length of dendritic growth cone filopodia were significantly affected in the presence of zerocalcium ACSF ( $p=0.52$ and $p=0.88$, respectively). Motility and lifetime of filopodia were similarly unaffected by zero-calcium ACSF in either dendritic shafts $(p>0.23)$ or growth cones $(p>0.32)$. The effects of zero-calcium ACSF on dendritic shaft filopodia density and length were reversible $\geq 30$ min after washing in normal ACSF containing $2 \mathrm{mM} \mathrm{CaCl}_{2}(p>0.28$ compared with control; $N=3$ successful washouts; $n=59$ ). D, Blocking ionotropic glutamate receptors for 15-30 min with $20 \mu \mathrm{M}$ CNQX and $40 \mu \mathrm{m} \mathrm{D}-\mathrm{APV}$ resulted in an $\sim 35 \%$ decrease in both the density $(p=0.006)$ and the turnover $(p=0.04)$ of shaft filopodia $(N=8 ; n=319)$ but had no effect on length, motility, or lifetime of shaft protrusions ( $p>0.15)$. CNQX/APV had no effect on growth cone filopodia ( $p>0.56 ; N=5 ; n=164$ ). These effects of CNQX/APV on dendritic shaft filopodia were reversed $30-60 \mathrm{~min}$ after washing out the drug ( $p>0.63$ compared with control; $N=4$ successful washouts; $n=116)$. E, PicoSpritzer application of $100-200 \mu \mathrm{m}$ glutamate resulted in an $\sim 75 \%$ increase in the length of shaft filopodia ( $p=0.004 ; N=5 ; n=251$ ) but did not significantly affect the other parameters $(p>0.25)$. A trend toward lower density of growth cone filopodia was also observed ( $p=0.055 ; N=2 ; n=76$ ). The histograms in $A-E$ reveal percentage differences compared with control dendrites imaged before the application of the drugs or zerocalcium ACSF. Error bars represent the SEM, using the number of experiments $(N)$, rather than the number of protrusions. ${ }^{*} p<0.05 ;{ }^{* *} p<0.01$.
} 


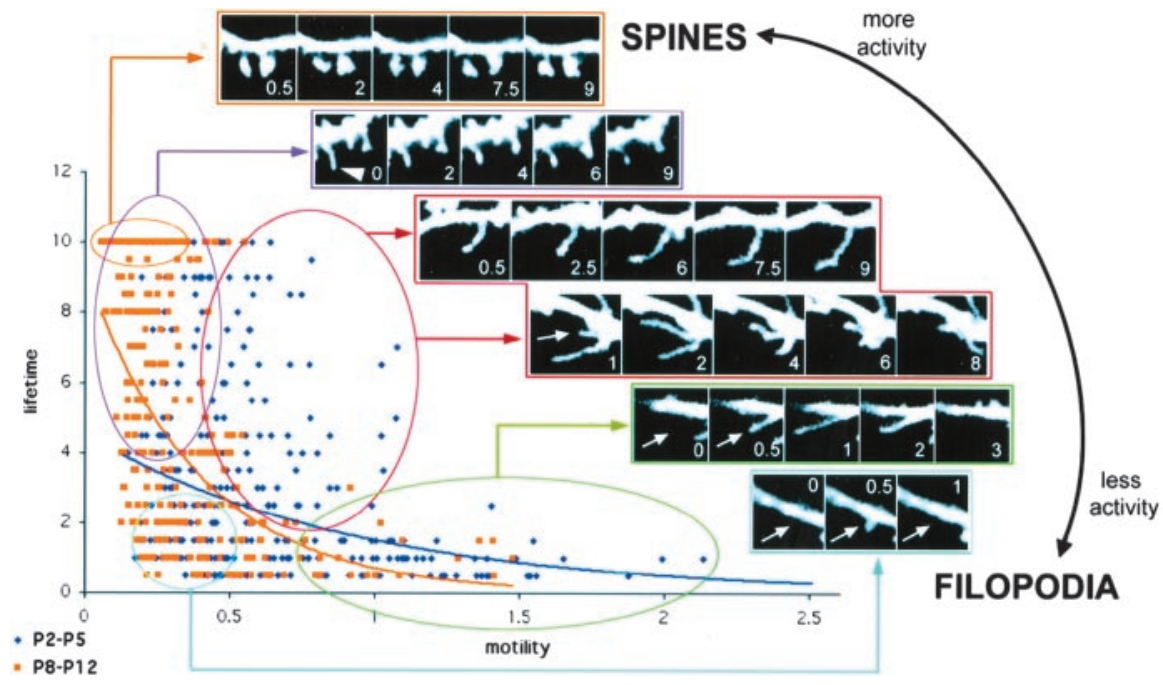

Figure 11. A continuum of dendritic shaft protrusions throughout early postnatal development. This graph plots motility on the $x$-axis against lifetime on the $y$-axis for a total of 342 individual shaft protrusions at P2-P5 (dark blue) and 333 individual shaft protrusions at P8 -P12 (light orange). The plot again demonstrates that at younger ages shaft protrusions are indeed more motile and have shorter lifetimes than at older ages, as was shown in Figure 5. The distribution of protrusions at either age is best fit by an exponential curve, but the characteristics of the distributions are different. At younger ages, as protrusion motility varies lifetime changes very little. The opposite is true at older ages when, as protrusions shift toward less motility, their lifetime increases considerably (and vice versa). On the basis of this distribution, a continuum of dendritic shaft protrusions can be discerned from the rapidly motile and transient filopodia (bottom right) to the relatively immobile and permanent dendritic spines (top left). We have arbitrarily defined several clusters of filopodia along this continuum. The insets show representative examples of imaged protrusions falling into several arbitrary categories of filopodia along this continuum, as well as an example of dendritic spines at P12. We speculate that neuronal activity regulates early dendritic protrusions such that, as neuronal activity increases throughout development, protrusions change along this continuum from filopodia to spine-like protrusions. Indeed, in more mature dendrites, some filopodia developed heads at their tips, thus resembling dendritic spines (arrowhead). Arrows indicate the position along the dendrite in which filopodia will appear in the next frame. Scale bar, $3 \mu \mathrm{m}$. Time stamps in white indicate minutes within a 10-min time-lapse movie.

dendritic filopodia are highly dynamic protrusions that are both temporally and spatially regulated. Our analysis demonstrates a developmental switch from highly motile and transient filopodia to relatively immobile and stable protrusions. Filopodia in growth cones behave differently than filopodia in shafts, and neuronal activity regulates dendritic shaft filopodia but not growth cone filopodia, suggesting distinct roles for filopodia in these two dendritic compartments. We propose the existence of a continuum of different types of dendritic shaft protrusions and that neuronal activity, and in particular glutamatergic transmission, may modulate this continuum. Although we focused on layer 5 pyramidal neurons from primary visual cortex, our observations in other cortical areas and layers suggest that this developmental, spatial, and activity regulation of dendritic filopodia represents a universal phenomenon.

\section{Methodological considerations}

It is possible that the slicing procedure may affect the parameters we studied (Kirov et al., 1999). To prevent any problems related to slicing, we limited our experiments to a specific window of time ( $1-4 \mathrm{hr}$ after slicing). Importantly, our numbers for density and length of early dendritic protrusions compare nicely with those derived from age-matched perfusion-fixed rat hippocampus analyzed with serial electron microscopy (C. Portera-Cailliau and K. Harris, unpublished observations), or with Golgi or biocytin stains (Linke et al., 1994). Thus, we feel that our slice data are a close reflection of what occurs in vivo. One should also remember that the alternative, in vivo imaging of spine or filopodial motility in anesthetized animals (Chen et al., 2000; Lendvai et al., 2000; Grutzendler et al., 2002; Trachtenberg et al., 2002), may be confounded by the effect of anesthetics, which appear to stop actin-based motility (Kaech et al., 1999). Furthermore, the surgery itself, as well as potential alterations in blood flow dynamics resulting from immobilization of the brain to reduce pulsation artifacts, could also alter protrusion motility.

\section{Growth cone filopodia: a role in} dendritic growth and branching One of the less predictable observations in this study was the finding that filopodia in dendritic growth cones are significantly more motile, longer, and more densely packed than filopodia in dendritic shafts. This suggests different roles for these two types of filopodia. Moreover, we found that dendritic growth cone filopodia are insensitive to neuronal activity blockade. This observation seems to contradict the synaptotropic hypothesis (Vaughn, 1989) that posits that dendritic growth cones grow toward sources of neuronal activity. Just as axonal growth cone filopodia are believed to guide axons to their target destinations, we propose that filopodia in dendritic growth cones also sense a chemical gradient to guide the dendrite, but this process is activity independent, at least in the short term $(<30 \mathrm{~min})$. Dendritic growth cones occur only transiently in development before $\mathrm{P} 5$, suggesting that dendritic tip growth and branching are essentially completed by that time. Additional dendritic growth after P5 must occur interstitially within the shafts, as the neuropil expands with the proliferation of glia, interneurons, and their processes. The longer lifetimes of tip protrusions after $\mathrm{P} 8$ suggests that when the dendritic tip growth is over, some very stable protrusions slowly replace the growth cone filopodia and anchor the dendrite tip in place.

\section{Shaft filopodia: a role in synaptogenesis or spinogenesis}

We report that neuronal activity regulates filopodia in dendritic shafts but not in growth cones. These results are consistent with the reported effects of activity blockade on spine density in cortical pyramidal neurons (Kirov et al., 1999) and in cerebellar Purkinje neurons (Bravin et al., 1999). The finding that ionotropic glutamate receptor antagonists result in a decrease in the density of shaft filopodia is similarly in agreement with previouswork on dendritic appendages on retinal ganglion cells (Wong et al., 2000). Given that synapses are known to exist along dendritic shaft filopodia (Saito et al., 1997; Fiala et al., 1998), one function of shaft filopodia may be to make early synapses. Our finding that some dendritic shaft filopodia elongate in response to glutamate supports this notion (similar findings were briefly noted in dissociated hippocampal neurons by Smith and colleagues (Smith and Jahr, 1992; Cornell-Bell et al., 1990). Thus, dendritic shafts may be seeking suitable synaptic partners by sensing glutamate gradients and, thus, extend filopodia toward nearby boutons to bridge the gap. At the same time, an explanation that would reconcile the somewhat contradictory TTX and CNQX/APV results remains elusive to us. One simple explanation is that TTX 
and zero-calcium conditions block neuronal activity globally, leading to alterations in a variety of both presynaptic and postsynaptic processes (including release of tropic substances other than glutamate). Thus, in the complete absence of neuronal activity, dendritic shafts broaden the search for axonal partners by increasing the numbers and lengths of filopodia. The opposite occurs during maturation when, as activity increases, dendritic protrusions stabilize (Fig. 11). And during development, a balanced basal level global activity is intermittently punctuated by local bursts of glutamate release that guide filopodia to their nearby targets.

It has been proposed that filopodia are precursors to spines, because of similarities in shape and the temporal order in which they appear during development (Ziv and Smith, 1996). One strong argument against this idea is that many adult neurons devoid of spines undergo a phase in development (at the time of early synaptogenesis), during which their dendrites bear numerous filopodia (Lund et al., 1977; Mason, 1983; Dvergsten et al., 1986; Ulfhake and Cullheim, 1988; Wong et al., 1992; Linke et al., 1994). Our current data also suggest that the predominant role of filopodia is not to give rise to spines, because the peak of filopodia expression occurs before P5, nearly 1 week before the first spine-like protrusions appear. We calculated the individual shaft protrusion turnover to be $\sim 1.2$ filopodia/min per 10 $\mu \mathrm{m}$ shaft segment of a $\mathrm{P} 2-\mathrm{P} 3$ dendrite. Because shaft filopodia density at that age is $\sim 4.5$ per $10 \mu \mathrm{m}$, we estimate that over a 10 -min movie, all the shaft filopodia would have recycled $\sim 2.5$ times over. Also, a P2-P3 neuron with 10 dendrites each measuring $30 \mu \mathrm{m}$ (a conservative estimate) would extend and retract over 50,000 shaft filopodia over the course of $1 \mathrm{~d}$. One can only conclude that the astronomical energy expenditure associated with this protrusion turnover in the first week of postnatal development is not aimed at the creation of spines, which occurs several days later. Instead, we favor the hypothesis that this intense filopodia activity corresponds to selective synaptogenesis with adequate presynaptic partners. This view is not incompatible, however, with the idea that, at older ages, some filopodia that become stabilized (protospines) could transform into spines (Fig. 11).

\section{A developmental continuum of early dendritic shaft protrusions: distinguishing filopodia from spines}

Despite gross morphological similarities, most of the evidence thus far suggests that spines and filopodia are different types of dendritic protrusions (for review, see Portera-Cailliau and Yuste, 2001). One of the strengths of our study is our fast temporal resolution because this allowed detailed measurements of the dynamic behavior of filopodia and spines. Thus, even though descriptive and static parameters (length, presence of head, etc.) are, unfortunately, still used routinely to distinguish spines from filopodia, we argue that lifetime and motility may be more accurate parameters to distinguish filopodia (lifetime, $\leq 3 \mathrm{~min}$; motility, $\geq 0.8 \mu \mathrm{m} / 30 \mathrm{sec}$ ) from spines (lifetime, $>8 \mathrm{~min}$; motility, $\leq 0.4 \mu \mathrm{m} / 30 \mathrm{sec}$ ). Indeed, plotting lifetime versus motility for all protrusions analyzed reveals a two-dimensional continuum of dendritic shaft protrusions, with some apparent clusters (Fig. 11). We have arbitrarily subdivided this spectrum and distinguished between several distinct populations of dendritic shaft protrusions. In the first $5 \mathrm{~d}$ of postnatal development, one can identify not only slow-moving protrusions with long lifetimes (red cluster) as well as the fastest-moving protrusions with short lifetimes (green cluster), but also slow-moving protrusions with short lifetimes (blue cluster). In the second week of postnatal development, the majority of protrusions are either nonmotile with long lifetimes (purple cluster) or slow-moving transient 
protrusions, whereas faster-moving protrusions are virtually nonexistent. We propose that neuronal activity modulates this continuum, such that as the brain matures and synaptic activity increases, protrusions shorten and become less motile.

\section{A dual model for filopodial function}

As mentioned previously, some investigators have argued that dendritic filopodia mediate dendritic growth and branching (Vaughn, 1989), whereas others have argued that filopodia give rise to dendritic spines (Ziv and Smith, 1996). On the basis of our data, we propose a solution to the existing controversy on the potential function of dendritic filopodia (Fig. 12). We would dissociate these two functions and argue that they are carried out by different populations of filopodia. In dendritic growth cones, like in axonal growth cones, filopodia would respond to guidance molecules (while being relatively insensitive to axonal cues or neuronal activity) and be responsible for dendritic growth and branching. At the same time, filopodia located in dendritic shafts would actively seek axonal terminals, presumably following glutamate gradients, to establish synaptic contacts with them and, at more mature stages, to further develop into spines. These two populations of filopodia might have different molecular structures and/or be linked to different molecular motors or second messenger cascades that reflect these diverging functions.

Our model predicts that the dynamic nature of both types of filopodia would be essential for their function, such that any approach to reduce or otherwise modify this motility could disrupt their ability to shape dendritic trees or form synapses. Additional investigations into clarifying the function of dendritic filopodia could be important to understand disorders such as mental retardation, autism, or epilepsy, characterized by abnormalities of dendritic protrusions (Fiala et al., 2001; PorteraCailliau and Yuste, 2001). Because filopodia probably mediate the process whereby dendrites make contact with their appropriate presynaptic partners, it follows that dysfunctional filopodia behavior and synaptogenesis could lead to aberrant circuits and neurological disturbances.

\section{References}

Bravin M, Morando L, Vercelli A, Rossi F, Strata P (1999) Control of spine formation by electrical activity in the adult rat cerebellum. Proc Natl Acad Sci USA 96:1704-1709.

Chen BE, Lendvai B, Nimchinsky EA, Burbach B, Fox K, Svoboda K (2000) Imaging high-resolution structure of GFP-expressing neurons in neocortex in vivo. Learn Mem 7:433-441.

Cornell-Bell AH, Thomas PG, Smith SJ (1990) The excitatory neurotransmitter glutamate causes filopodia formation in cultured hippocampal astrocytes. Glia 3:322-334.

Dailey ME, Smith SJ (1996) The dynamics of dendritic structure in developing hippocampal slices. J Neurosci 16:2983-2994.

Denk W, Svoboda K (1997) Photon upmanship: why multiphoton imaging is more than a gimmick. Neuron 18:351-357.

Difiglia M, Pasik P, Pasik T (1980) Early postnatal development of the monkey neostriatum: a Golgi and ultrastructural study. J Comp Neurol 190:303-331.

Dunaevsky A, Tashiro A, Majewska A, Mason CA, Yuste R (1999) Developmental regulation of spine motility in mammalian CNS. Proc Natl Acad Sci USA 96:13438-13443.

Dvergsten CL, Hull CD, Levine MS, Adinolfi AM, Buchwald NA (1986) Postnatal differentiation and growth of cat entopeduncular neurons. A transient spiny period associated with branch elongation. Brain Res 389:239-251.

Feng G, Mellor RH, Bernstein M, Keller-Peck C, Nguyen QT, Wallace M, Nerbonne JM, Lichtman JW, Sanes JR (2000) Imaging neuronal subsets in transgenic mice expressing multiple spectral variants of GFP. Neuron 28:41-51.

Fiala JC, Feinberg M, Popov V, Harris KM (1998) Synaptogenesis via den- dritic filopodia in developing hippocampal area CA1. J Neurosci 18:8900-8911.

Fiala JC, Spacek J, Harris KM (2001) Dendritic spine pathology: cause or consequence of neurological disorders? Brain Res Brain Res Rev 39:29-54.

Fischer M, Kaech S, Knutti D, Matus A (1998) Rapid actin-based plasticity in dendritic spines. Neuron 20:847-854.

Grutzendler J, Kasthuri N, Gan WB (2002) Long-term dendritic spine stability in the adult cortex. Nature 420:812-816.

Jontes JD, Smith SJ (2000) Filopodia, spines, and the generation of synaptic diversity. Neuron 27:11-14.

Kaech S, Brinkhaus H, Matus A (1999) Volatile anesthetics block actinbased motility in dendritic spines. Proc Natl Acad Sci USA 96:10433-10437.

Kirov SA, Harris KM (1999) Dendrites are more spiny on mature hippocampal neurons when synapses are inactivated. Nat Neurosci 2:878-883.

Kirov S, Sorra K, Harris K (1999) Slices have more synapses than perfusionfixed hippocampus from both young and mature rats. J Neurosci 19:2876-2886.

Lendvai B, Stern E, Chen B, Svoboda K (2000) Experience-dependent plasticity of dendritic spines in the developing rat barrel cortex in vivo. Nature 404:876-881.

Linke R, Soriano E, Frotscher M (1994) Transient dendritic appendages on differentiating septohippocampal neurons are not the sites of synaptogenesis. Brain Res Dev Brain Res 83:67-78.

Lo DC, McAllister AK, Katz LC (1994) Neuronal transfection in brain slices using particle-mediated gene transfer. Neuron 13:1263-1268.

Lund JS, Boothe RG, Lund RD (1977) Development of neurons in the visual cortex (area 17) of the monkey (Macaca nemestrina): a Golgi study from fetal day 127 to postnatal maturity. J Comp Neurol 176:149-188.

Majewska A, Tashiro A, Yuste R (2000a) Regulation of spine calcium compartmentalization by rapid spine motility. J Neurosci 20:8262-8268.

Majewska A, Yiu G, Yuste R (2000b) A custom-made two-photon microscope and deconvolution system. Pflügers Arch Eur J Physiol 441:398-409.

Maletic-Savatic M, Malinow R, Svoboda K (1999) Rapid dendritic morphogenesis in CAl hippocampal dendrites induced by synaptic activity. Science 283:1923-1927.

Mason CA (1983) Postnatal maturation of neurons in the cat's lateral geniculate nucleus. J Comp Neurol 217:458-469.

Morest DK (1969) The differentiation of cerebral dendrites: A study of the post-migratory neuroblast in the medial nucleus of the trapezoid body. Z Anat Entwicklungsgesch 128:271-289.

Nikolenko V, Nemet B, Yuste R (2003) A two-photon and secondharmonic microscope. Methods 30:3-15.

Parnass Z, Tashiro A, Yuste R (2000) Analysis of spine morphological plasticity in developing hippocampal pyramidal neurons. Hippocampus 10:561-568.

Peters A, Kaiserman-Abramof IR (1970) The small pyramidal neuron of the rat cerebral cortex: the perikaryon, dendrites and spines. J Anatom 127:321:356.

Portera-Cailliau C, Yuste R (2001) On the function of dendritic filopodia. Rev Neurol 33:1158-1566.

Purpura DP (1975) Dendritic differentiation in human cerebral cortex: normal and aberrant development patterns. In: Advances in neurology (Kreutzberg GW, ed), pp 91-116. New York: Raven.

Ramón y Cajal S (1888) Estructura de los centros nerviosos de las aves. Rev Trim Histol Norm Pat 1:1-10.

Ramón y Cajal S (1891) Sur la structure de l'ecorce cerebrale de quelques mamiferes. La Cellule 7:124-176.

Ramón y Cajal S (1899) La textura del sistema nerviosa del hombre y los vertebrados. Madrid: Moya (Primera Edicion).

Ramón y Cajal S (1934) Les preuves objectives de l'unite anatomique des cellules nerveuses. In: Revista Trimestral Micrografica. Travaux du laboratoire de recherches biologiques de l'Universite de Madrid, Madrid, pp $1-137$.

Saito Y, Song WJ, Murakami F (1997) Preferential termination of corticorubral axons on spine-like dendritic protrusions in developing cat. J Neurosci 17:8792-8803.

Smith SJ, Jahr CE (1992) Rapid induction of filopodial sprouting by application of glutamate to hippocampal neurons. In: The nerve growth cone 
(Letourneau PC, Kater SB, Macagno ER, eds), pp 19-27. New York: Raven.

Trachtenberg JT, Chen BE, Knott GW, Feng G, Sanes JR, Welker E, Svoboda $\mathrm{K}$ (2002) Long-term in vivo imaging of experience-dependent synaptic plasticity in adult cortex. Nature 420:788-794.

Ulfhake B, Cullheim S (1988) Postnatal development of cat hind limb motoneurons. II: In vivo morphology of dendritic growth cones and the maturation of dendrite morphology. J Comp Neurol 278:88-102.

Vaughn J, Henrikson C, Grieshaber J (1974) A quantitative study of synapses on motor neuron dendritic growth cones in developing mouse spinal cord. J Cell Biol 60:664-672.
Vaughn JE (1989) Fine structure of synaptogenesis in the vertebrate central nervous system. Synapse 3:255-285.

Wong ROL, Yamawaki RM, Shatz CL (1992) Synaptic contacts and the transient dendritic spines of developing retinal ganglion cells. Eur J Neurosci 4:1387-1397.

Wong WT, Faulkner-Jones BE, Sanes JR, Wong ROL (2000) Rapid dendritic remodeling in the developing retina: dependence on neurotransmission and reciprocal regulation by Rac and Rho. J Neurosci 20:5024-5036.

Ziv N, Smith S (1996) Evidence for a role of dendritic filopodia in synaptogenesis and spine formation. Neuron 17:91-102. 